LMU-ASC 40/06

HD-THEP-06-10

hep-th/0606047

\title{
On the modified KKLT procedure: a case study for the $\mathbf{P}_{11169}[18]$ model
}

\author{
Gottfried Curio $^{a}$ and Vera Spillner ${ }^{b}$ \\ ${ }^{a}$ Arnold-Sommerfeld-Center for Theoretical Physics \\ Department für Physik, Ludwig-Maximilians-Universität München \\ Theresienstraße 37, 80333 München, Germany \\ ${ }^{b}$ Institut für Theoretische Physik, Universität Heidelberg \\ Philosophenweg 16, 69120 Heidelberg, Germany
}

\begin{abstract}
We probe the existence of supersymmetric vacua of the type IIB orientifold of the elliptic Calabi-Yau space $\mathbf{P}_{11169}[18]$ where generically two complex structure moduli $z_{i}$, the dilaton $\tau$ and the two Kähler moduli $T_{i}$ are stabilized by fluxes and gaugino condensates. The usual KKLT procedure, which integrates out the complex structure moduli and the dilaton, actually has to be modified, such that one keeps the dependence on $\tau$. We derive explicitely the resulting effective superpotential $W_{\text {eff }}(\tau)$ for the dilaton for various flux combinations. As this is actually a non-holomorphic quantity one must properly work with the $G$-function. The remaining SUSY equations for $\tau$ and the $T_{i}$ can be resolved explicitely.
\end{abstract}




\section{Introduction}

The issue of moduli stabilization in superstring theory gained new interest when it was observed [1], [2] that by adding 3-form fluxes, inducing a superpotential $W_{\text {flux }}$, in the type IIB theory on a Calabi-Yau $X$ the complex structure moduli $z_{i}$ and the dilaton $\tau$ can be frozen. In a corresponding orientifold model, reducing $4 D$ space-time supersymmetry to $N=1$, the presence of orientifold planes leads to cancellation of $D$-brane charges against the effects of $D 3 / D 7$-branes and 3-form fluxes in the tadpole cancellation condition.

KKLT [3] combined this with a non-perturbative superpotential $W_{n p}$ describing gaugino condensation [4] (or Euclidean D3-instantons [5]) such that the remaining Kähler moduli $T_{i}$ could be stabilized also. Here, for warped $D 7$-branes, the non-abelian gauge theory on a stack of $D 7$-branes must allow for a non-vanishing gaugino condensate; for Euclidean D3-brane instantons the number of fermionic zero-modes on the world-volume is relevant (here the fluxes have a subtle impact on the relevant count [6]).

More precisely, the scenario of KKLT amounts (working at supergravity level at large $\tau$ and $T_{i}$ ) to a two-step procedure where $z_{i}$ and $\tau$ have been fixed by fluxes first, leading just to a constant effective superpotential contribution $W_{0}$, and in a second step, in the resulting effective theory, the Kähler moduli $T_{i}$ are stabilized (this decoupling procedure assumes that the $z_{i}$ and $\tau$ are much heavier than the $T_{i}$ so that they can be integrated out first). The resulting supersymmetric $A d S$-vacuum was then uplifted to a non-supersymmetric $d S$-vacuum by adding anti $D 3$-branes (for whose stability the (mass) $)^{2}$ eigenvalues of the fixed scalars should be already positive in the $A d S$-vacuum and not just negative and fulfilling the Breitenlohner-Freedman bound).

In [7] stability conditions were derived (for a susy $A d S$-vacuum with stabilized moduli) in a more general case than the decoupling limit of KKLT. There only the $z_{i}$ (if heavy enough) were integrated out, leading to an effective superpotential contribution $W_{\text {eff }}(\tau)$

$$
W_{\text {flux }}\left(z_{i}, \tau\right)=A\left(z_{i}\right)+\tau B\left(z_{i}\right) \stackrel{D_{i} W_{\text {flux }}=0}{\longrightarrow} W_{\text {eff }}(\tau)
$$

(define for later $\gamma:=\tau_{2} W_{e f f}^{\prime \prime} / W_{e f f}^{\prime}$ ). There as ansatz for the Kähler potential is used

$$
K^{(T)}(T)+K(\tau)=-3 \log (T+\bar{T})-\log (\tau-\bar{\tau})
$$

The supersymmetric stationary points fulfill $D_{i} W=0$. Their nature is determined by the second derivatives $V^{\prime \prime}$ of the scalar potential of the combined superpotential $W=$ $W_{\text {eff }}(\tau)+C e^{-a T}$ (here in principle the $C$ was also $z_{i}$-dependent, but is set constant for now): this matrix splits into 'real' and 'imaginary' (axionic) parts $V_{0 M}^{\prime \prime}$ and $V_{0 A}^{\prime \prime}$ and the stability condition for $V$ from the matrix determinants (at large $T$ ) is $|\gamma|>1$. 
In [8] it was pointed out that this program should be properly enhanced to take into account two points. First, the Kähler potential $K\left(z_{i}\right)$ of the complex structure moduli, more precisely the effective version $K_{\text {eff }}(\tau)=K\left(z_{i}(\tau)\right)$ of it arising after integrating out the $z_{i}$, has to be added to $K(\tau)=-\ln (\tau-\bar{\tau})$. Secondly, as the conditions $D_{i} W_{\text {flux }}=0$ for integrating out the $z_{i}$ are (because of the covariant derivative involving the Kähler potential) actually non-holomorphic, it turns out that the effective superpotential $W_{\text {eff }}(\tau)$ arising from $W_{\text {flux }}\left(\tau, z_{i}\right)$ is also actually a non-holomorphic quantity. Therefore one should compute the scalar potential in the form $V=e^{G}\left(G^{i \bar{\jmath}} G_{i} G_{\bar{\jmath}}-3\right)$ where

$$
G=K(\tau)+K_{e f f}(\tau)+K(T)+\ln \left|W_{e f f}+W^{(T)}\right|^{2}
$$

and the $z_{i}$ should be integrated out by $G_{i}=0$ or equivalently (for $\left.W \neq 0\right) D_{i}\left(W_{\text {flux }}+\right.$ $\left.W^{(T)}\right)=0$, leading actually to $z_{i}(\tau, T)$ instead of just $z_{i}(\tau)$. This is described in sect. 5 .

Just as in the case of KKLT this program now should be carried out for concrete examples. We have choosen the elliptic Calabi-Yau $X=\mathbf{P}_{11169}(18)$ and its corresponding orientifold model for which the KKLT program was carried through [9], cf. also [10], [11], [12]. For some examples of choices of fluxes we derive here $W_{\text {eff }}$ and $K_{\text {eff }}$ in completely explicit form. For one fully worked out case we find that no supersymmetric solutions exist. For the procedures cf. sect. 6. Here we were just searching for SUSY solutions; more generally one would search for non-SUSY stationary points of the resulting $V$.

In one concrete example we take the flux combination (where we specialise to $c=3 d$ )

$$
\left(e_{R}^{a} \mid m_{R}^{a}\right)=(0, g, 0 \mid 0,-a,-b) \quad, \quad\left(e_{N S}^{a} \mid m_{N S}^{a}\right)=(0,0,0 \mid 0, c, d)
$$

which gives for $W_{f l u x}=g F_{1}+a z_{1}+b z_{2}+\tau\left(c z_{1}+d z_{2}\right)$ the explicit expression

$$
W_{\text {flux }}\left(z_{i}, \tau\right)=g\left(-\frac{1}{2}\left(3 z_{1}+z_{2}\right)^{2}+\frac{3}{2}\left(3 z_{1}+z_{2}\right)+\frac{17}{4}\right)+(a+\tau c) z_{1}+(b+\tau d) z_{2}
$$

The Kähler potential of the complex structure moduli $z_{i}$ is given by (where $y_{k}=\operatorname{Im} z_{k}$ )

$$
K\left(z_{1}, z_{2}\right)=-\ln \left[y_{1}\left(3 y_{1}^{2}+3 y_{1} y_{2}+y_{2}^{2}\right)+i \xi\right]
$$

The fourfold we work on following [9] has a base $B_{3}$, itself $\mathbf{P}_{\mathbf{z}}^{\mathbf{1}}$ fibered over $B_{2}=\mathbf{P}^{\mathbf{2}}$; the latter is embedded as divisor $r$ and $r_{\infty}$ in $B_{3}$ at $z=0$ and $z=\infty$, respectively, and has a $G_{2}$ singularity along $r$ and an $E_{6}$ or $E_{8}$ singularity along $r_{\infty}$. With the volumes $\tau_{1}:=\tau_{r}=\frac{1}{2} t_{1}^{2}$ and $\tau_{2}:=\tau_{r_{\infty}}=\frac{1}{2}\left(t_{1}+6 t_{2}\right)^{2}$ of the divisors $r$ and $r_{\infty}$ a superpotential

$$
W^{(T)}=C_{1} e^{-2 \pi a_{1} \mathcal{T}_{1}}+C_{2} e^{-2 \pi a_{2} \mathcal{T}_{2}}
$$

is generated (with the complexified 4-cycle-volumes $\mathcal{T}_{j}=\tau_{j}+i \theta_{j}$ where $\theta_{i}=-\int_{r_{(\infty)}} C_{4}$ ). For the Kähler form $J=t_{1} L+t_{2} r_{\infty}$ (cf. below) one has the Kähler potential (so $\tau_{2}>\tau_{1}$ )

$$
K\left(t_{i}\right)=-2 \ln \left(\tau_{2}^{3 / 2}-\tau_{1}^{3 / 2}\right)
$$


In section 2 we consider the $N=2$ theory from which the orientifold model is derived. We give the prepotential, the Kähler potential and the period vector for the complex structure moduli, leading to a concrete expression of $W_{\text {flux }}$. In section 3 we give the $N=1$ orientifold theory with its Kähler potential. In section 4 we recall the KKLT procedure for the model. In section 5 we show how one works for $W_{\text {eff }}$ non-holomorphic actually with the $G$ function. In section 6 we derive $W_{\text {eff }}\left(\tau, \mathcal{T}_{k}\right)$ for various flux combinations and give a complete analytical treatment of the remaining SUSY conditions for $\tau$ and the $\mathcal{T}_{k}$.

\section{The $N=2$ parent theory on the Calabi-Yau $X_{3}$}

We consider the elliptic Weierstrass fibration $\pi: X \rightarrow B_{2}$ over $B_{2}=\mathbf{P}^{\mathbf{2}}$. Some data of such elliptic Calabi-Yau threefolds (with section $\sigma$ ) over $B_{2}$ are (by adjunction) [15]

$$
\begin{aligned}
\sigma^{2} & =-c_{1} \sigma \\
c_{2}(Z) & =12 c_{1} \sigma+c_{2}+11 c_{1}^{2}
\end{aligned}
$$

One has $h^{1,1}(X)=2$ from the divisors $\sigma$ (the embedded base of the fibration) and $L:=\pi^{-1} l$ where $l$ is the line in $B_{2}$. Furthermore $c_{3}(X)=-60 c_{1}^{2}$ gives $h^{2,1}(X)=272$.

Classical intersection numbers on $Z$

With $H:=\sigma+3 L$ and $L$ as divisors one finds using (2.1)

$$
H^{3}=9, \quad H^{2} L=3, \quad H L^{2}=1, \quad L^{3}=0
$$

Furthermore one gets from (2.2) that that

$$
c_{2}(Z) \cdot L=36 \quad, \quad c_{2}(Z) \cdot H=108+\left(-12 c_{1}^{2}+c_{2}+11 c_{1}^{2}\right)=102
$$

Kähler potential

Let us give the corresponding Kähler potential for the Kähler form $\tilde{\mathcal{J}}:=t_{1} H+\tilde{t}_{2} L$

$$
e^{-K}=V=\frac{1}{6}\left(9 t_{1}^{3}+9 t_{1}^{2} \tilde{t}_{2}+3 t_{1} \tilde{t}_{2}^{2}\right)
$$

Remark: This is the Kähler potential in the basis adapted to the prepotential given below. Later, when we consider the orientifold $N=1$ theory derived from the $N=2$ Calabi-Yau theory, the relevant divisors to consider will be suitable quotients related to $\sigma$ (then called $r$ ) and $L$. For this reason let us also give here already the Kähler potential for the Kähler form $\mathcal{J}^{\prime}:=t_{1} \sigma+t_{2}^{\prime} L$, i.e. with intersection numbers

$$
\begin{aligned}
\sigma^{3}=9, \quad \sigma^{2} L & =-3, \quad \sigma L^{2}=1, \quad L^{3}=0 \\
e^{-K}=V & =\frac{1}{6}\left(9 t_{1}^{3}-9 t_{1}^{2} t_{2}^{\prime}+3 t_{1}\left(t_{2}^{\prime}\right)^{2}\right)
\end{aligned}
$$




\section{The prepotential and the periods}

Expressing the middle homology and cohomology in terms of a symplectic basis, i.e. a basis of 3-cycles $A^{a}$ and $B_{b}$ with $A^{a} \cap B_{b}=-B_{b} \cap A^{a}=\delta_{b}^{a}$ and $A^{a} \cap A^{b}=B_{a} \cap B_{b}=0$ and a basis of 3 -forms $\alpha_{a}$ and $\beta^{b}$ (where $a, b=0,1, \ldots, h^{2,1}$; let $n=h^{2,1}+1$ )

$$
\int_{A^{b}} \alpha_{a}=-\int_{B_{a}} \beta^{b}=\delta_{a}^{b}, \quad \int_{X} \alpha_{a} \wedge \beta^{b}=-\int_{X} \beta^{b} \wedge \alpha_{a}=\delta_{a}^{b}
$$

Such a symplectic basis is only defined up to $S p(2 n, \mathbf{Z})$ transformations, as these preserve the symplectic intersection form. The periods are defined via the holomorphic 3 -form $\Omega$

$$
\int_{A_{a}} \Omega=X^{a}, \quad \int_{B^{b}} \Omega=\mathcal{F}_{a}
$$

The periods are collected in the period vector, $\Pi=\left(X^{0}, \ldots, X^{n}, F_{0}, \ldots, F_{n}\right)$. This is a function of the complex structure moduli $z^{i}=X^{i} / X_{0}\left(i=1, \ldots, h^{2,1}\right)$ and inherits the holomorphic freedom of $\Omega$ and is defined up to holomorphic rescalings $\Omega \rightarrow f\left(z_{i}\right) \Omega$.

Here the prepotential $\mathcal{F}$ is given by (with $\xi^{\prime}=\frac{\zeta(3)}{2(2 \pi i)^{3}} \chi(X) \approx-1.3 i$ and $q_{i}=e^{2 \pi i z_{i}}$ )

$$
\begin{aligned}
\mathcal{F} & =-\frac{C_{i j k}}{3 !} \frac{X^{i} X^{j} X^{k}}{X^{0}}+\frac{A_{i j}}{2} X^{i} X^{j}+c_{i} X^{i} X^{0}-\xi^{\prime}\left(X^{0}\right)^{2}+f(q)\left(X^{0}\right)^{2} \\
& =\left(X^{0}\right)^{2} \mathcal{F}=\left(X^{0}\right)^{2}\left(-\frac{C_{i j k}}{3 !} z^{i} z^{j} z^{k}+\frac{1}{2} A_{i j} z^{i} z^{j}+c_{i} z^{i}-\xi^{\prime}+f(q)\right)
\end{aligned}
$$

where $C_{i j k}=\int J_{i} \wedge J_{j} \wedge J_{k}=D_{i} \cap D_{j} \cap D_{k}$ are the classical intersection numbers and $c_{i}=\frac{1}{24} \int c_{2}(X) J_{i}$ (the quadratic coeffecients $A_{i j}$ are unphysical and not completely fixed).

In our case this becomes (we take later $A_{11}=9 / 2, A_{12}=3 / 2, A_{22}=0$ with [16], [9])

$$
\mathcal{F}=-\frac{1}{6}\left(9 z_{1}^{3}+9 z_{1}^{2} z_{2}+3 z_{1} z_{2}^{2}\right)+\frac{9}{4} z_{1}^{2}+\frac{3}{2} z_{1} z_{2}+\frac{17}{4} z_{1}+\frac{3}{2} z_{2}-\xi^{\prime}
$$

With this prepotential the period vector becomes (where $F_{a}=\partial F / \partial X^{a}$ and $\mathcal{F}_{i}=$ $\partial \mathcal{F} / \partial z^{i}$ for the prepotential $F\left(X^{a}\right)$ in homogeneouos coordinates $\left(a=0,1, \ldots, h^{2,1}\right)$ and $\mathcal{F}\left(z^{i}\right)$ in inhomogeneouos coordinates $z^{i}=X^{i} / X^{0}\left(i=1, \ldots, h^{2,1}\right)$, respectively $)$

$$
\Pi=\left(\begin{array}{c}
X^{0} \\
X^{i} \\
F_{0} \\
F_{i}
\end{array}\right)=X^{0}\left(\begin{array}{c}
1 \\
z^{i} \\
2 \mathcal{F}-z^{i} \mathcal{F}_{i} \\
\mathcal{F}_{i}
\end{array}\right)=X^{0}\left(\begin{array}{c}
1 \\
z^{i} \\
\frac{C_{i j k}}{3 !} z^{i} z^{j} z^{k}+c_{i} z^{i}-2 \xi^{\prime}+\ldots \\
-\frac{C_{i j k}}{2} z^{j} z^{k}+A_{i j} z^{k}+c_{i}+\ldots
\end{array}\right)
$$

The Kähler potential for the complex structure moduli space is given by

$$
\begin{aligned}
K\left(z_{i}\right) & =-\ln \left(i \int \Omega \wedge \bar{\Omega}\right)=-\ln \left(-i \Pi^{\dagger} \cdot \Sigma \cdot \Pi\right) \\
& =-\ln \left(i\left|X^{0}\right|^{2}\left(\left(z_{i}-\bar{z}_{i}\right)\left(\mathcal{F}_{i}+\overline{\mathcal{F}}_{i}\right)-2(\mathcal{F}-\overline{\mathcal{F}})\right)\right)
\end{aligned}
$$

with $\Sigma=\left(\begin{array}{cc}0 & \mathbf{1}_{\mathbf{n}} \\ -\mathbf{1}_{\mathbf{n}} & 0\end{array}\right)$. Further one has in the dilaton sector $K(\tau)=-\ln (-i(\tau-\bar{\tau}))$. 
One calculates explicitly (with $z_{k}=x_{k}+i y_{k} ; \xi:=-\xi^{\prime}$ )

$$
K\left(z_{1}, z_{2}\right)=-\ln \left(y_{1}\left(3 y_{1}^{2}+3 y_{1} y_{2}+y_{2}^{2}\right)+i \xi\right)
$$

(up to an additive constant). Compare the Kähler potential (2.14) for the complex structure moduli (expressed in the $y_{k}$ ) with the Kähler potential (2.5) for the Kähler moduli (expressed in $t_{1}=\operatorname{Re} T_{1}, \tilde{t}_{2}=\operatorname{Re} T_{2}$ and with respect to $H$ and $L$ )

$$
K\left(t_{1}, \tilde{t}_{2}\right)=-\ln \left(\frac{1}{2}\left(3 t_{1}^{3}+3 t_{1}^{2} \tilde{t}_{2}+t_{1} \tilde{t}_{2}^{2}\right)\right)
$$

Restricting the consideration of the complex structure moduli to two of them (as recalled below) one gets the same Kähler potential as for the Kähler moduli by mirror symmetry.

Note that $Z$ has the (weighted) projective embedding as hypersurface $\mathbf{P}_{11169}[18]$

$$
x_{1}^{18}+x_{2}^{18}+x_{3}^{18}+x_{4}^{3}+x_{5}^{2}-18 \psi x_{1} x_{2} x_{3} x_{4} x_{5}-3 \phi x_{1}^{6} x_{2}^{6} x_{3}^{6}=0
$$

Here actually only 2 of the full set of 272 deformations are displayed. The group $\Gamma=$ $\mathbf{Z}_{6} \times \mathbf{Z}_{18}$ is a symmetry of the moduli space and fixes the two-parameter subspace in (2.16). One is lead to this specific Calabi-Yau by the mirror construction and the periods of $\Gamma$-invariant cycles are its six periods. One proceeds to find flux-vacua by working in the $\Gamma$-invariant part of the moduli space (turning on only invariant fluxes); i.e. one turns on fluxes just on these cycles and has $D_{i} W=0$ in non-invariant directions as the resulting superpotential and Kählerpotential are invariant. Effectively it is thus consistent to set all other moduli to zero and work only on the moduli in (2.16) and their associated fluxes.

\subsection{The flux superpotential}

In terms of the periods, the flux-superpotential is ${ }^{1}$

$$
\begin{aligned}
W_{G} & =\int_{X} G_{3} \wedge \Omega=(2 \pi)^{2} \alpha^{\prime}\left(\left(e_{R}^{a}-\tau e_{N S}^{a}\right) \cdot \Pi_{3+a}-\left(m_{R}^{a}-\tau m_{N S}^{a}\right) \cdot \Pi_{a}\right) \\
& =(2 \pi)^{2} \alpha^{\prime}\left(\sum_{a=0}^{k}\left(e_{R}^{a}-\tau e_{N S}^{a}\right) F_{a}-\left(m_{R}^{a}-\tau m_{N S}^{a}\right) z_{a}\right)
\end{aligned}
$$

where the integral vectors of fluxes along the cycles occur as precoefficients. More precisely the fluxes $H_{R}$ and $H_{N S}$ are elements of $H^{3}(Z, \mathbf{Z})$ quantised as follows

$$
e_{R / N S}^{a}=\frac{1}{(2 \pi)^{2} \alpha^{\prime}} \int_{A^{a}} H_{R / N S} \in \mathbf{Z}, \quad\left(m_{R / N S}\right)_{a}=\frac{1}{(2 \pi)^{2} \alpha^{\prime}} \int_{B_{a}} H_{R / N S} \in \mathbf{Z}
$$

This amounts to a D3-charge carried by the fluxes

$$
N_{\text {flux }}=L=\frac{1}{2}\left(e_{R}^{a}, m_{R}^{a}\right) \cdot \Sigma \cdot\left(e_{N S}^{a}, m_{N S}^{a}\right)^{t}=\frac{1}{2} \sum_{a=0}^{k} e_{R}^{a} m_{N S}^{a}-m_{R}^{a} e_{N S}^{a}
$$

\footnotetext{
${ }^{1}$ note that for two three-forms one has $\int \phi \wedge \chi=\sum_{a} \int_{A^{a}} \phi \int_{B_{a}} \chi-\int_{B_{a}} \phi \int_{A^{a}} \chi$
} 


\section{The $N=1$ theory and the orientifold limit of the fourfold}

Let $\pi: X_{4} \rightarrow B_{3}$ denote the projection of the elliptic fibration of a Calabi-Yau fourfold in $F$-theory. We consider the case that $B_{3}$ is $\mathbf{P}^{\mathbf{1}}$-fibered over the base $B_{2}$. In the orientifold limit a $D 4$ singularity is realized.

In the type IIB string theory one describes $B_{3}$ as quotient by a holomorphic involution of a Calabi-Yau threefold $Z$, branched at the $B_{3}$-location of the singularities of the elliptic fibration of $X_{4}\left(D_{4}\right.$ singularities corresponding to an $O 7$-plane and four coincident $D 7$ branes). $D$-branes are introduced to cancel the $R R$ tadpoles produced by the orientifold plane. If $Z$ is given as a hypersurface in an ambient $\mathbf{P}^{\mathbf{4}}$ this involution might be given by $z_{1} \rightarrow-z_{1}$.

The dilaton is the complex structure modulus of the elliptic $F$-theory fibre.

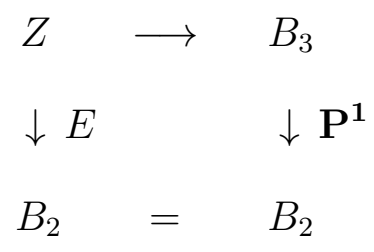

$B_{3}$ inherits the Kähler and complex structure moduli which are even and odd, respectively, under the involution.

Classical intersection numbers on $B_{3}$

The $\mathbf{P}^{1}$-fibration of $B_{3}$ over $B_{2}$ can be described as projectivization $\mathbf{P}(Y)$ of a vector bundle $Y=\mathcal{O} \oplus \mathcal{T}$ with $\mathcal{T}$ a line bundle over $B_{2}$.

Let $\mathcal{O}(1)$ denote a line bundle on the total space which restricts on each $\mathbf{P}^{\mathbf{1}}$ fibre to the corresponding line bundle over $\mathbf{P}^{\mathbf{1}}$ and let $r=c_{1}(\mathcal{O}(1))$ and $t=c_{1}(\mathcal{T})$ so that the cohomology of $B_{3}$ is generated over $B_{2}$ by $r$ with the relation $r(r+t)=0$ (as the two section are disjoint: one has $r=r_{0}$ the divisor given by $B_{2}$ itself inside $B_{3}$, at the zerosection of the $\mathbf{P}^{\mathbf{1}}$-fibration; the section at infinity is given by $r_{\infty}=r+t$ ). Concretely take $B_{2}=\mathbf{P}^{2}$ and $t=6 l$. Here we denote the line in $B_{2}$ by $l$ and its preimage in $B_{3}$ by $L=\mathbf{F}_{\mathbf{6}}$ (one has $L r^{2}=\left(\left.r\right|_{L}\right)^{2}=(l)_{L}^{2}=-6$; furthermore $L r_{\infty}^{2}=6$ ).

One has the following intersection numbers in $B_{3}$ (the first three relations are obvious)

$$
L^{3}=0, \quad L^{2} r=L^{2} r_{\infty}=1, \quad L r_{\infty}^{2}=6, \quad r_{\infty}^{3}=r^{3}=36
$$


For this note first that by adjunction

$$
\left.c\left(B_{3}\right)\right|_{D}=\left.(1+D)\left(1+c_{1}(D)+c_{2}(D)\right)\right|_{D}
$$

one has the relation

$$
c_{2}\left(B_{3}\right) \cdot D=D^{2} \cdot c_{1}\left(B_{3}\right)-D^{3}+\int_{D} c_{2}(D)
$$

Further one has for the $\mathbf{P}^{\mathbf{1}}$-fibration defined by $t$ that (unspecified $c_{i}$ refer always to $B_{2}$ )

$$
\begin{aligned}
& c_{1}\left(B_{3}\right)=2 r+c_{1}+t \\
& c_{2}\left(B_{3}\right)=2 c_{1} r+c_{1} t+c_{2}
\end{aligned}
$$

again from adjunction $c\left(B_{3}\right)=\left(1+c_{1}+c_{2}\right)(1+r)(1+r+t)$. (3.4) now gives $r^{3}=36$ as

$$
\begin{aligned}
c_{2}\left(B_{3}\right) \cdot r & =\left(-c_{1} t+c_{2}\right) r=-c_{1} t+3 \\
r^{2} \cdot c_{1}\left(B_{3}\right)-r^{3}+\int_{r} c_{2}(r) & =-r\left(c_{1} t+t^{2}\right)+r^{3}+3=-c_{1} t-36+r^{3}+3
\end{aligned}
$$

\section{The Kähler potential}

One can now compute the Kähler potential for the Kähler form $J^{\prime}:=t_{1} L+t_{2}^{\prime} r$. Alternatively and equivalently one can consider $r_{\infty}$ instead of $r$

$$
J=t_{1} L+t_{2} r_{\infty}
$$

One gets for the divisor volumes $\tau_{r_{(\infty)}}=r_{(\infty)} J^{2} / 2$ and for the total volume $V=J^{3} / 6$

$$
\begin{aligned}
\tau_{1}:=\tau_{r} & =\frac{1}{2} t_{1}^{2}, \quad \tau_{2}:=\tau_{r_{\infty}}=\frac{1}{2}\left(t_{1}+6 t_{2}\right)^{2} \\
V & =\frac{1}{6}\left(3 t_{1}^{2} t_{2}+18 t_{1} t_{2}^{2}+36 t_{2}^{3}\right)=\frac{1}{36}\left(\left(t_{1}+6 t_{2}\right)^{3}-t_{1}^{3}\right)=\frac{\sqrt{2}}{18}\left(\tau_{2}^{3 / 2}-\tau_{1}^{3 / 2}\right)(3
\end{aligned}
$$

implying that $\tau_{2}>\tau_{1}$ in the Kähler cone. With this one gets the Kähler potential

$$
K\left(t_{i}\right)=-2 \ln V
$$

Remark: For direct comparison with the Kähler potential (2.7) of the $N=2$ parent theory let us also give here the Kähler potential w.r.t. $J^{\prime}$ (i.e. $\sigma$ above becomes $r$ below)

$$
e^{-\frac{1}{2} K}=V=\frac{1}{12}\left(9\left(2 t_{2}^{\prime}\right)^{3}-9\left(2 t_{2}^{\prime}\right)^{2} t_{1}+3\left(2 t_{2}^{\prime}\right) t_{1}^{2}\right)
$$




\section{Integrating out $z_{i}$ and $\tau$ and working with $W_{0}$}

The stabilisation of $\tau$ and the $z_{i}$ is achieved from the conditions

$$
D_{i} W=\partial_{i} W+\left(\partial_{i} K\right) W=0 \quad, \quad D_{\tau} W=\partial_{\tau} W+\left(\partial_{\tau} K\right) W=0
$$

In the given example one finds a stationary point for the values ${ }^{2}[9] z_{1}=i=z_{2}, \tau=3 i$. This vacuum of ${ }^{3} W=0$ is reached for the following flux combination of $N_{\text {flux }}=352$

$$
\begin{gathered}
\left(e_{R}^{a} \mid m_{R}^{a}\right)=(20,0,0 \mid 0,-69,-28) \quad, \quad\left(e_{N S}^{a} \mid m_{N S}^{a}\right)=(0,-4,0 \mid 49,18,6) \\
W=69 z_{1}+28 z_{2}+20 F_{0}-\tau\left(-49-18 z_{1}-6 z_{2}-4 F_{1}\right) \\
=30 z_{1}^{3}+30 z_{1}^{2} z_{2}+10 z_{1} z_{2}^{2}+154 z_{1}+58 z_{2}+40 \xi+\tau\left(-2\left(3 z_{1}+z_{2}\right)^{2}+27 z_{1}+9 z_{2}+66\right)
\end{gathered}
$$

\subsection{The Kähler stabilizing superpotential}

[9] find no divisors leading to an instanton contribution in the superpotential following the necessary condition $\chi(D)=1$ for the arithmetic genus $[5] .{ }^{4}$ One computes $\chi\left(\pi^{-1} C\right)=$ $-C^{2} c_{1}\left(B_{3}\right) / 2$ [13]. Recall now that the base $B_{3}$ of $X_{4}$ was $\mathbf{P}_{\mathbf{z}}^{\mathbf{1}}$ fibered over $B_{2}=\mathbf{P}^{\mathbf{2}}$ and one had divisors $C=r$ and $r_{\infty}$ in $B_{3}$ as sections of this $\mathbf{P}_{\mathbf{z}}^{\mathbf{1}}$ fibration at $z=0$ and $z=\infty$, respectively. For the projection $p r: B_{3} \rightarrow B_{2}$ (with $t=n l$, say) one finds for the $p r$-horizontal divisors (in $\left.B_{3}\right) r$ and $r_{\infty}$ and for the $p r$-vertical divisor $p r^{-1} P$ (for $P=a l$ )

$$
\chi\left(\pi^{-1} r_{0 / \infty}\right)=\frac{1}{2} t\left( \pm c_{1}-t\right)=\frac{1}{2} n( \pm 3-n) \quad, \quad \chi\left(\pi^{-1} p r^{-1} P\right)=-P^{2}=-a^{2}
$$

So one has to rely solely on gauge type divisors (where contributions are induced from gaugino condensation; cf. also [14]). The concrete fourfold we work with following [9] has actually a $G_{2}$ (not $\left.D_{4}\right)$ singularity along $r$ and an $E_{6}$ or $E_{8}$ singularity along $r_{\infty}$. This leads to values of $\chi\left(X_{4}\right) / 24$ of 273,129 , respectively (note however that $N_{\text {flux }}=352$ ).

With the volumes $\tau_{1}:=\tau_{r}$ and $\tau_{2}:=\tau_{r_{\infty}}$ of the divisors $r$ and $r_{\infty}$ a superpotential

$$
W=W_{0}+C_{1} e^{-2 \pi a_{1} \mathcal{T}_{1}}+C_{2} e^{-2 \pi a_{2} \mathcal{T}_{2}}
$$

is generated where we introduced the complexified 4-cycle-volumes $\mathcal{T}_{j}=\tau_{j}+i \theta_{j}$ with $\theta_{i}=-\int_{r_{(\infty)}} C_{4}$. Assuming $W_{0}$ real (and $C_{i}=1$ ) and that the two exponential terms should equal approximately $W_{0}$ at the critical point, they should be also approximately equal there. In view of $\tau_{1}<\tau_{2}$ this means that $a_{1}>a_{2}$. For some numerical solutions in the set-up above, where $a_{1}=1 / 4$ and $a_{2}=1 / 12$ or $1 / 30$, with $W_{0}=10^{-5}$ or $10^{-30}$ cf. [9].

\footnotetext{
${ }^{2}$ with instanton corrections consistently ignored for $\operatorname{Im} z_{1}, \operatorname{Im} z_{2} \geq 1$

${ }^{3}$ With the first instanton correction one finds a near-by vacuum with $e^{K}|W|^{2}=1.379 \times 10^{-4}$.

${ }^{4}$ As actually $t=6 l$ all $\chi$ values in (4.2) are negative, so the flux influence can not change them to 1 .
} 


\section{$5 \quad$ Integrating out the $z_{i}$ and working with $K_{\text {eff }}(\tau)$}

The action for (gauge neutral) chiral mutiplets can be expressed in terms of a single real function $G$. The scalar potential becomes (with $G_{i \bar{\jmath}}=\partial_{i} \partial_{\bar{\jmath}} G$ and $G_{i}=\partial_{i} G, G^{i}=G^{i \bar{\jmath}} G_{\bar{\jmath}}$ )

$$
V=e^{G}\left(G^{i \bar{\jmath}} G_{i} G_{\bar{\jmath}}-3\right)=e^{K}\left(K^{i \bar{j}} D_{i} W \overline{D_{\bar{j}}} \bar{W}-3 W \bar{W}\right)
$$

The index $i$ runs over all chiral superfields, here the moduli and the dilaton. $G$ can be, and usually is, (ambiguously) split as $G=K+\ln |W|^{2}$ into a real Kähler potential $K(z, \bar{z})$ and a holomorphic superpotential $W(z)$. The supersymmetry conditions are $D_{i} W=W_{i}+K_{i} W=W G_{i}=0$. So, for $W \neq 0$, the supersymmetry conditions are in terms of $G$ given by $G_{i}=0$.

\section{On the stability analysis in general}

The stability analysis is based on the eigenvalues of the mass matrix. In the AdS case, one has to check whether the BF bound is satisfied. But in the case where one adds a term such that the vacuum energy becomes positive, one has to make sure that all eigenvalues of the mass matrix are actually positive. This must be done in a field basis where the kinetic terms are canonically normalized. However the eigenvalues in both bases are not the same but their signs are the same. If they are all positive in the original basis, they are also positive in the canonical basis (so a negative eigenvalue signals an instability).

To parallelize the treatment of the stability criterion of [7] let us recall also here the mass matrix, now in terms of $G$. First, the stationarity condition $\partial_{k} V=0$ becomes

$$
\partial_{k} V=e^{G}\left(\left(G^{i} G_{i}-3\right) G_{k}+\left(G^{i} \nabla_{k} G_{i}+G_{k}\right)\right)
$$

At the miminum one gets the $(k l)$-symmetric (as $G_{i \bar{\jmath}}$ is a Kähler metric) expression ${ }^{5}$

$$
\begin{aligned}
\left.\partial_{l} \partial_{k} V\right|_{d V=0} & =e^{G}\left(\nabla_{l} G_{k}+\nabla_{k} G_{l}+\left(G^{i} G_{i}-3\right) \nabla_{l} G_{k}+G^{i} \nabla_{l} \nabla_{k} G_{i}+\left(G_{l}+G^{i} \nabla_{l} G_{i}\right) G_{k}\right) \\
& =e^{G}\left(\nabla_{l} G_{k}+\nabla_{k} G_{l}+G^{i} \nabla_{l} \nabla_{k} G_{i}+\left(G^{i} G_{i}-3\right)\left(\nabla_{l} G_{k}-G_{l} G_{k}\right)\right)
\end{aligned}
$$

For the mixed derivatives one finds (using $\left.\nabla_{\bar{l}} \nabla_{k} G_{i}=R_{\overline{l k i}}{ }^{j} G_{j}\right)^{6}$

$\left.\partial_{\bar{l}} \partial_{k} V\right|_{d V=0}=e^{G}\left(G_{k \bar{l}}+\left(G^{i} G_{i}-3\right) G_{k \bar{l}}+G_{k}\left(G_{\bar{l}}+G^{\bar{\jmath}} \nabla_{\bar{l}} G_{\bar{\jmath}}\right)+\nabla_{k} G_{i} \nabla_{\bar{l}} G^{i}+R_{\bar{l} k i}{ }^{j} G_{j} G^{i}\right)(5.4)$

For a SUSY vacuum with $G_{i}=0$ one has the simple expressions

$$
\begin{aligned}
& \left.\partial_{l} \partial_{k} V\right|_{\text {susy vacuum }}=-e^{G} \partial_{k} \partial_{l} G \\
& \left.\partial_{\bar{l}} \partial_{k} V\right|_{\text {susy vacuum }}=e^{G}\left(-2 G_{k \bar{l}}+G^{i \bar{\jmath}} \partial_{k} G_{i} \partial_{\bar{l}} G_{\bar{\jmath}}\right)
\end{aligned}
$$

\footnotetext{
${ }^{5}$ For Minkowski vacua one is left with the first three terms (the last of these is missing in [17]).

${ }^{6}$ For Minkowski vacua one finds the first term and the last two terms, cf. [17].
} 


\subsection{Restricted preconsideration: the $\left(z_{i}, \tau\right)$-sector}

There are two equivalent ways to handle the integrating-out procedure. Either one eliminates the $z_{i}$ in $G$ (cf. below) and then operates, after reinserting them, with a $G_{e f f}$; alternatively one derives $W_{e f f}^{n o n-h o l o}(\tau)$ from $D_{i} W_{\text {flux }}\left(z_{i}, \tau\right)=0$ first and takes then, working in the $G$-formalism, $W_{\text {eff }}^{\text {hol }}(\tau)=1$ and $K_{\text {tot }}(\tau)=K(\tau)+K\left(z_{i}(\tau)\right)+\ln \left|W_{\text {eff }}^{\text {non-holo }}\right|^{2}$ and the corresponding $G_{e f f}=K_{t o t}$; for this cf. [8] and the example treated by us below. Concretely from the expressions given earlier one gets then the $z_{i}$-elimination conditions

$$
D_{z_{i}} W=\partial_{z_{i}} W+\partial_{z_{i}} K W=0
$$

These equations allow to eliminate the $z_{i}$ in $W_{f l u x}\left(z_{i}, \tau\right)$ and to get an effective superpotential $W_{\text {eff }}^{\text {non-holo }}(\tau)$ (this replaces the earlier constant $W_{0}$ when also $\tau$ was integrated out $)^{7}$. One gets then, in this seond way, the complete Kähler potential in $\tau$ as follows

$$
\begin{aligned}
K(\tau) & =-\ln (\tau-\bar{\tau}) \\
K_{\text {eff }}(\tau) & =K\left(z_{i}(\tau)\right) \\
K_{\text {tot }}(\tau) & =K(\tau)+K_{\text {eff }}(\tau)+\ln \left|W_{\text {eff }}^{\text {non-holo }}(\tau)\right|^{2}
\end{aligned}
$$

Concerning the first procedure, as explained in the introduction, our strategy will be to solve the supersymmetry conditions for all the fields which appear in $G$. We first eliminate the $z_{i}$ by solving, for a suitable choice of fluxes, $\partial_{z_{i}} G=0$ where

$$
G=K(\tau)+K\left(z_{i}\right)+\ln \left|W_{f l u x}\left(z_{i}, \tau\right)\right|^{2}
$$

The equations can be solved for the $z_{i}$ and the solutions, which depend on $\tau$, produce $G_{\text {eff }}(\tau)$ when reinserted into $G$. Two points are worth mentioning here. First, since we are working with the real function $G$, questions of holomorphicity of the superpotential do not arise. This is an issue, since the SUSY conditions $D_{z_{i}} W=0$ are not holomorphic and would lead to a non-holomorphic $W_{\text {eff }}$. Secondly, one also has to insert the solutions $z_{i}(\tau)$ into $K\left(z_{i}\right)$ and not merely into $W_{f l u x}$.

Note that, for $W \neq 0$, both procedures are equivalent as the respective elimination conditions are $G_{i}=0$ and $D_{i} W=0$.

Before we come to the main issue of this note, the consideration of the case with two Kähler parameters, we give first the procedure in the generic one parameter case, following [7] but including $K\left(z_{i}(\tau)\right)$ and $\ln \left|W_{\text {eff }}^{\text {non-holo }}(\tau)\right|^{2}$. We will see that including the $\mathcal{T}$-sector leads to a slight change in the procedure.

\footnotetext{
${ }^{7}$ the fluxes chosen earlier which lead to the concrete expressions for $W_{\text {flux }}$ gave a minimum at $z_{1}=$ $z_{2}=i, \tau=3 i$ in the procedure where $\tau$ is also integrated out; we can, of course, choose other fluxes.
} 


\subsection{Full $\left(z_{i}, \tau, \mathcal{T}\right)$-sector: the generic one parameter case}

In the generic set-up with one Kähler parameter one would start in analogy to [7] with

$$
W=W_{\text {flux }}\left(z_{i}, \tau\right)+W^{(\mathcal{T})}=A\left(z_{i}\right)+\tau B\left(z_{i}\right)+C e^{-a \mathcal{T}}
$$

with the Kähler potential (where $K(\mathcal{T})=-2 \ln (\mathcal{T}+\overline{\mathcal{T}})^{3 / 2}$ )

$$
K=-\ln (\tau-\bar{\tau})+K\left(z_{i}\right)+K(\mathcal{T})
$$

Integrating out the $z_{i}$ by $G_{i}=0$ resp. $G_{i} \cdot W=D_{i} W=D_{i}\left(W_{\text {flux }}+C e^{-a \mathcal{T}}\right)=0$ gives

$$
D_{i} W=A_{i}+\tau B_{i}+K_{i}\left(W_{\text {flux }}+C e^{-a \mathcal{T}}\right)=0
$$

where compared with the previous (5.7) the exponentially suppressed perturbation term $K_{i} W^{(\mathcal{T})}$ has arisen. Therefore now the previous $z_{i}=z_{i}(\tau)$ becomes here $z_{i}=z_{i}(\tau, \mathcal{T})=$ $z_{i}(\tau)+\delta W^{(\mathcal{T})}+\epsilon \bar{W}^{(\overline{\mathcal{T}})}+\ldots$, cf. [8]. So one is led to use a $W_{\text {eff }}^{\text {holo }}(\tau, \mathcal{T}) \equiv 1$ and the Kähler potential

$$
\begin{aligned}
G_{\text {eff }}=K_{\text {eff }}= & -\ln (\tau-\bar{\tau})+K\left(z_{i}(\tau, \mathcal{T})\right)-3 \ln (\mathcal{T}+\overline{\mathcal{T}}) \\
& +\ln \left|A\left(z_{i}(\tau, \mathcal{T})\right)+\tau B\left(z_{i}(\tau, \mathcal{T})\right)+C e^{-a \mathcal{T}}\right|^{2}
\end{aligned}
$$

Note that to get $G_{\text {eff }}$ in (5.15), it would have been again equivalent (for $W \neq 0$ ) to start with the full $G^{\text {full }}=-\ln (\tau-\bar{\tau})+K\left(z_{i}\right)+K(\mathcal{T})+\ln \left|W_{\text {flux }}\left(z_{i}, \tau\right)+W^{(\mathcal{T})}\right|^{2}$, solve there $G_{i}^{\text {full }}=0$ for the $z_{i}(\tau, \mathcal{T})$ and reinserting into $G^{\text {full }}$, for one has $D_{i} W_{\text {flux }}=W G_{i}^{\text {full }}$.

Note in passing that the procedure described is not the same as doing the previous integrating-out procedure just in the $\left(z_{i}, \tau\right)$-sector with $W_{e f f}^{\text {holo }}(\tau) \equiv 1$ and just adding the $\mathcal{T}$-sector. This leads to the following point: in the two-step analysis of KKLT all moduli of $W_{\text {flux }}\left(z_{i}, \tau\right)$ were integrated out; this left behind just a $W_{0}$ when afterwards the further $W^{(\mathcal{T})}$ was included. However, as [7] pointed out, this procedure may not be justified as the masses of $\tau$ and $\mathcal{T}$ can be comparable; so in [7] just the $z_{i}$ were integrated out, leaving a $W_{\text {eff }}(\tau)$ behind instead of the constant $W_{0}$. This procedure, as outlined in [8] and described also below, has to be supplemented, at least, with the inclusion of $K\left(z_{i}(\tau)\right)$ and a proper treatment of $W_{e f f}^{\text {non-holo }}(\tau)$. As we described above, even including these points, there would be still a remnant of the linkage of $\tau$ with the $z_{i}$, if $z_{i}(\tau)$ would be assumed to be just a function of $\tau$ (what would arise from integrating it out while considering just $W_{\text {flux }}\left(z_{i}, \tau\right)$, and including $W^{(\mathcal{T})}(\mathcal{T})$ only afterwards) instead of determining a $z_{i}(\tau, \mathcal{T})$ from the full $W=W_{\text {flux }}\left(z_{i}, \tau\right)+W^{(\mathcal{T})}(\mathcal{T})$. 


\section{The two-parameter example}

Take the flux components with the general full magnetic sector

$$
\left(e_{R}^{a} \mid m_{R}^{a}\right)=(0, g, h \mid-e,-a,-b) \quad, \quad\left(e_{N S}^{a} \mid m_{N S}^{a}\right)=\left(0,0,0 \mid e^{\prime}, c, d\right)
$$

Concerning the two special magnetic fluxes $e$ and $e^{\prime}$ which contribute in $W_{\text {flux }}$ only by adding a constant $E=e+e^{\prime} \tau$, which is independent of the complex structure moduli, we will set at first $e=e^{\prime}=0$. We will employ the following notation (and $z_{k}=x_{k}+i y_{k}$ )

$$
\begin{array}{rl}
A:=a+\tau c=A_{1}+i A_{2} & B:=b+\tau d=B_{1}+i B_{2} \\
Z:=3 z_{1}+z_{2}=X+i Y & y:=y_{2} \\
p:=\frac{a-3 b}{3}, & q:=\frac{c-3 d}{3} \\
W^{(\mathcal{T})}=\sum_{k=1}^{2} W^{k} & =\sum_{k=1}^{2} C_{k} e^{-a_{k} \mathcal{T}_{k}}
\end{array}
$$

We will always stick to the case $q=0$, i.e. $c=3 d$ (note that this was also satisfied in the flux-choice (4.1)); we exclude the case $c=0=d$ where things collapse trivially.

We will then write the two SUSY conditions $D_{z_{i}} W=0$ which allow us to eliminate the $z_{i}$ and write $W_{\text {eff }}(\tau)$. The way we proceed to do this in practice amounts to writing $W_{\text {flux }}$ (with the help of the SUSY conditions) purely as function of $\tau$ and only one of the four real variables $X, Y, x_{2}, y$, concretely either $Y$ or $y$, where the latter furthermore depends in a simple polynomial way on $\tau$.

We then proceed and include the $\mathcal{T}_{k}$-sector. For that purpose we generalize the above set-up first to include fluxes $m_{R}^{0}=-e, m_{N S}^{0}=e^{\prime}$ and the corresponding contribution

$E=e+e^{\prime} \tau=E_{1}+i E_{2}$ in $W_{\text {flux }}$. The latter will be used then to mimic the $W^{(\mathcal{T})}$. This will be described more fully below in our main example, the case II. In total the following cases will be described

$$
\begin{array}{cc}
\text { CASE I } & g=0, \quad h=0 \\
\text { CASE II } & g \neq 0, \quad h=0 \\
\text { CASE III } & g \neq 0, h=-3 g
\end{array}
$$

We will get an explicit expression for $W_{\text {eff }}$ in all 3 cases. In the case II, which constitutes our main example, we will proceed and analyse the SUSY conditions completely in closed analytical form and show that no SUSY solution exists. As the main issue here is the explicit derivation of $W_{e f f}$ and the concrete analytical treatment of the ensuing SUSY conditions we give this in some detail so that one can follow the line of derivation. 


\subsection{The case I: $g=h=0$}

We will at first assume that $g=h=0$. Although this leads to the somewhat degenerate case of $L=0$ we mention this case first as some equations appear here first in a somewhat simpler form than in our main example case II. This leads to

$$
W_{\text {flux }}=a z_{1}+b z_{2}+\tau\left(c z_{1}+d z_{2}\right)=A z_{1}+B z_{2}=\frac{A}{3} Z-p z_{2}
$$

which gives as elimination equations (where $z_{k}=x_{k}+i y_{k}$ )

$$
\begin{aligned}
D_{z_{1}} W=0 \Longrightarrow 2 i \frac{A}{3}=\frac{\frac{1}{3}\left(3 y_{1}+y_{2}\right)^{2}}{y_{1}\left(3 y_{1}^{2}+3 y_{1} y_{2}+y_{2}^{2}\right)+i \xi}\left(A z_{1}+B z_{2}\right) \\
D_{z_{2}} W=0 \Longrightarrow 2 i B=\frac{y_{1}\left(3 y_{1}+2 y_{2}\right)}{y_{1}\left(3 y_{1}^{2}+3 y_{1} y_{2}+y_{2}^{2}\right)+i \xi}\left(A z_{1}+B z_{2}\right)
\end{aligned}
$$

This yields (for $Y \neq 0$, as is needed to keep things nontrivial) the compatibility equation

$$
\frac{A}{3}\left(Y^{2}-y^{2}\right)=B Y^{2}
$$

As the $y_{k}$ are real and $A_{2} / 3=B_{2}$ the imaginary part here gives $y=0$, with $p Y^{2}=0$ as remaining relation; one gets therefore also $p=0$ and the expressions $W_{\text {flux }}=\frac{A}{3} Z$ and

$$
W_{\text {flux }}=2 i \frac{A}{3} \frac{3 y_{1}^{3}+i \xi}{3 y_{1}^{2}}
$$

The resulting relation $Z=2 i \frac{3 y_{1}^{3}+i \xi}{3 y_{1}^{2}}$ gives $X=0$ and $Y=2 \frac{Y^{3}+9 i \xi}{3 Y^{2}}$ or

$$
\begin{aligned}
Y & =\sqrt[3]{2 \cdot 9 i \xi} \quad\left(\Longleftarrow Y^{3}+3 C Y^{2}-2 \cdot 9 i \xi=0 \quad \text { with } C:=0\right) \\
W_{e f f}(\tau) & =\frac{A}{3} Z=i A y_{1}=i(a+c \tau) \sqrt[3]{\frac{2}{3} i \xi}
\end{aligned}
$$

(Note that, if one would force now also the third condition (usual KKLT procedure)

$$
D_{\tau} W_{\text {flux }}=0 \Longrightarrow 2 i d Z=\frac{1}{\tau_{2}}\left(A z_{1}+B z_{2}\right)
$$

one would get $2 i d \tau_{2}=\frac{a+c \tau}{3}$, i.e. $\tau=-\frac{a}{c}$ with $\tau_{2}=0$, i.e. no (physical) solution.)

Although this example is somewhat degenerate $(L=0)$ let us proceed and include the $\mathcal{T}_{k}$-sector. So we generalize the above to include fluxes $m_{R}^{0}=-e, m_{N S}^{0}=e^{\prime}$ and the corresponding contribution $E=e+e^{\prime} \tau=E_{1}+i E_{2}$ in $W_{\text {flux }}$. Then the trivial $C$ above changes to an $E$-dependent $\tilde{C}$ (we use now the abbreviated notation $\mathcal{A}:=A / 3$ ). 
One gets $W_{\text {flux }}=\mathcal{A} Z+E$ and equating with (6.10) gives for the real and imaginary part

$$
\begin{aligned}
X & =-\frac{\mathcal{A}_{1} E_{1}+\mathcal{A}_{2} E_{2}}{\mathcal{A}_{1}^{2}+\mathcal{A}_{2}^{2}} \\
Y^{3}+3 \tilde{C} Y^{2}-2 \cdot 9 i \xi & =0 \quad\left(\tilde{C}:=\frac{\mathcal{A}_{1} E_{2}-\mathcal{A}_{2} E_{1}}{\mathcal{A}_{1}^{2}+\mathcal{A}_{2}^{2}}\right)
\end{aligned}
$$

With this cubic determination equation one gets for the effective Kählerpotential (up to an additive constant) and the effective superpotential (with $E:=W^{(\mathcal{T})}$ ) and $G$-function

$$
\begin{aligned}
K_{\text {eff }}\left(\tau, \mathcal{T}_{k}\right) & =-\ln \left(Y^{3}-y^{3}+9 i \xi\right)=-2 \ln Y-\ln \mathcal{B} \\
W_{\text {eff }}\left(\tau, \mathcal{T}_{k}\right) & =\mathcal{A}(X+i Y)+E=\frac{2 i}{3} \mathcal{A} \frac{Y^{3}+9 i \xi}{Y^{2}}=i \mathcal{A B} \\
G_{\text {eff }}\left(\tau, \mathcal{T}_{k}\right) & =K_{\text {eff }}\left(\tau, \mathcal{T}_{k}\right)+\ln \left|W_{\text {eff }}\left(\tau, \mathcal{T}_{k}\right)+W^{(\mathcal{T})}\right|^{2}-\ln (\tau-\bar{\tau})+K\left(\mathcal{T}_{k}\right)
\end{aligned}
$$

Here we use the following notation

$$
\begin{gathered}
\mathcal{B}=Y+\tilde{C} \quad, \quad s_{k}=(-1)^{k} \frac{3}{2} \frac{t_{k}^{1 / 2}}{t_{2}^{3 / 2}-t_{1}^{3 / 2}} \\
\Gamma=\mathcal{B} \frac{\mathcal{A} \overline{\mathcal{A}} \mathcal{B}+\left(\mathcal{A}_{1} \operatorname{Im} W-\mathcal{A}_{2} \operatorname{Re} W\right)}{\left(\mathcal{A}_{1} \mathcal{B}+\operatorname{Im} W\right)^{2}+\left(-\mathcal{A}_{2} \mathcal{B}+\operatorname{Re} W\right)^{2}}, \quad \Gamma^{\prime}=\frac{-i \overline{\mathcal{A}} \mathcal{B}+\overline{W^{(\mathcal{T})}}}{\left(\mathcal{A}_{1} \mathcal{B}+\operatorname{Im} W\right)^{2}+\left(-\mathcal{A}_{2} \mathcal{B}+\operatorname{Re} W\right)^{2}}
\end{gathered}
$$

Note that one still has $y=0$ as before. Furthermore $Y \neq 0$ and the effective Kähler potential gives from $Y^{3}-y^{3}+9 i \xi=Y^{3}+9 i \xi=\frac{3}{2} Y^{2}(Y+\tilde{C})$ that also $\mathcal{B} \neq 0$.

Furthermore one has the following relations ( $l$ stands for $k$ or $\tau$ )

$$
\begin{gathered}
Y_{l}(Y+2 \tilde{C})=-\tilde{C}_{l} Y \\
\tilde{C}_{k}=3 i \frac{a_{k} W^{k}}{2 A} \quad, \quad \tilde{C}_{\tau}=3 i \frac{c W^{(\mathcal{T})}}{2 A^{2}} \\
Y_{l}=\tilde{C}_{l} \frac{-Y}{Y+2 \tilde{C}} \quad, \quad \mathcal{B}_{l}=\tilde{C}_{l} \frac{2 \tilde{C}}{Y+2 \tilde{C}}
\end{gathered}
$$

From $G_{\text {eff }}$ (in the following denoted by $G$ ) one gets as SUSY conditions

$$
\begin{aligned}
& G_{k}=-2 \frac{Y_{k}}{Y}+(2 \Gamma-1) \frac{\mathcal{B}_{k}}{\mathcal{B}}-a_{k} W^{k} \Gamma^{\prime}+s_{k}=0 \\
& G_{\tau}=-2 \frac{Y_{\tau}}{Y}+(2 \Gamma-1) \frac{\mathcal{B}_{\tau}}{\mathcal{B}}+i \frac{c}{3} \mathcal{B} \Gamma^{\prime}+\frac{i}{2 \tau_{2}}=0
\end{aligned}
$$

For a full treatment of such a set of equations we refer to the case II below. As the case I is somewhat degenerate $(L=0)$ we are content here to derive $W_{\text {eff }}$ (for more details on the eliminations following from the detailed treatment of the SUSY equations, along the lines of the complete procedure as in case II, cf. the appendix). 


\subsection{The case II: $g \neq 0, h=0$}

For an example where $L=c g / 2 \neq 0$ take (again in the case $A_{2}=3 B_{2}$, i.e. ${ }^{8} c=3 d$ )

$$
\left(e_{R}^{a} \mid m_{R}^{a}\right)=(0, g, 0 \mid 0,-a,-b) \quad, \quad\left(e_{N S}^{a} \mid m_{N S}^{a}\right)=(0,0,0 \mid 0, c, d)
$$

which gives (with $A=A_{1}+i A_{2}:=a+c \tau, B=B_{1}+i B_{2}:=b+d \tau$ )

$$
W_{\text {flux }}=g F_{1}+a z_{1}+b z_{2}+\tau\left(c z_{1}+d z_{2}\right)=g\left(-\frac{1}{2} Z^{2}+\frac{3}{2} Z+\frac{17}{4}\right)+A z_{1}+B z_{2}
$$

We use the following abbreviations (for $g \neq 0$ )

$$
C:=\frac{A_{1}+\frac{9}{2} g}{3 p}, \quad \beta=\frac{B_{2}}{g}
$$

Useful relations are

$$
\begin{aligned}
A z_{1}+B z_{2} & =\frac{A}{3} Z-p z_{2} \\
9 y_{1}\left(3 y_{1}^{2}+3 y_{1} y_{2}+y_{2}^{2}\right) & =Y^{3}-y_{2}^{3}
\end{aligned}
$$

One gets as elimination equations $D_{z_{1}} W=0$ and $D_{z_{2}} W=0$

$$
\begin{aligned}
& {\left[\frac{A}{3}-g\left(Z-\frac{3}{2}\right)\right]=\frac{3}{2 i} \frac{Y^{2}}{Y^{3}-y^{3}+9 i \xi}\left(g F_{1}+A z_{1}+B z_{2}\right)} \\
& {\left[B-g\left(Z-\frac{3}{2}\right)\right]=\frac{3}{2 i} \frac{Y^{2}-y^{2}}{Y^{3}-y^{3}+9 i \xi}\left(g F_{1}+A z_{1}+B z_{2}\right)}
\end{aligned}
$$

(recall we denote $y_{2}$ just by $y$ ). Now the imaginary and real part of the compatibility equation

$$
\left[\frac{A}{3}-g\left(Z-\frac{3}{2}\right)\right]\left(Y^{2}-y^{2}\right)=\left[B-g\left(Z-\frac{3}{2}\right)\right] Y^{2}
$$

yield for $y \neq 0$ and $g \neq 0$ the eliminations

$$
Y=\beta \quad, \quad X=\frac{p}{g}\left(C-\frac{\beta^{2}}{y^{2}}\right)
$$

Finally we have to solve the imaginary and real part of (6.31), say, which gives, noting that the big fraction on the right hand side is real and that (by explicit evaluation)

$$
\operatorname{Im} W_{\text {flux }}=p(C \beta-y)
$$

finally the cubic equation

$$
y^{3}-3 C \beta y^{2}+2\left(\beta^{3}+9 i \xi\right)=0
$$

\footnotetext{
${ }^{8}$ We assume $c \neq 0 \neq d$ to retain a proper $\tau$-dependence in $W_{\text {flux }}$ after integrating out the $z_{i}$.
} 
Let us remark on the cubic equation occuring here.

1) (6.36) is of course easily solved in general. If one would be interested only in purely imaginary $\tau$ (note that $\tau_{2}=s=\operatorname{Re} S$ under $\tau=$ : $i S$, where $\tau=\tau_{1}+i \tau_{2}$ ), i.e. $A_{1}=$ $a, B_{1}=b$, and furthermore in the special case $C=0$, i.e. $a=-\frac{9}{2} g$, then

$$
W_{e f f}\left(\tau_{2}\right)=-i p y=i\left(b+\frac{3}{2} g\right)\left(-2\left(\frac{d^{3}}{g^{3}} \tau_{2}^{3}+9 i \xi\right)\right)^{1 / 3}
$$

2) In the general case one gets for $r:=y-C \beta$ with $W_{\text {eff }}=-i p r$ the equation

$$
r^{3}+\operatorname{Pr}+Q=r^{3}-3 C^{2} \beta^{2} r+2\left(\left(1-C^{3}\right) \beta^{3}+9 i \xi\right)=0
$$

(note $\beta, C, i \xi, P, Q \in \mathbf{R}$ ) with the three solutions given by the Cardano formula where one solution is always real and all three are real for $0 \geq D:=(Q / 2)^{2}+(P / 3)^{3}$.

Now let us come back to the use of this information for our main goal, whic is to derive the supersymmetry conditions from the effective $G$-function. One now has a $K_{\text {eff }}(\tau)=K\left(z_{i}(\tau)\right)$ which can be written in various equivalent ways

$$
\begin{aligned}
K_{e f f}(\tau) & =-\ln \left(\beta^{3}+9 i \xi-y^{3}\right)-\ln \frac{4 i}{9} \\
& =-\ln \left(\beta^{3}+9 i \xi-C \beta y^{2}\right)-\ln \frac{4 i}{3} \\
& =-\ln y^{2}-\ln (y-C \beta)-\ln \frac{-2 i}{3}
\end{aligned}
$$

giving in total (up to the constant $-\ln \frac{-2 i}{3}$ )

$$
K_{t o t}(\tau)=-\ln (\tau-\bar{\tau})-2 \ln y-\ln (y-C \beta)
$$

Note that, in contrast to the original piece $-\ln (\tau-\bar{\tau})$, the new, full $K_{\text {tot }}(\tau)$ depends not just on $\tau_{2}$ as the coefficient $C$ (which determines together with $\beta$ the $y$ ) depends on $\tau_{1}$.

Because of its non-holomorphicity one gets (for $r \in \mathbf{R}$ ) from $W_{\text {eff }}$ where

$$
W_{e f f}(\tau)=i p(\beta C-y)
$$

the further contribution (up to the constant $2 \ln p$ )

$$
\ln \left|W_{e f f}^{\text {non-holo }}(\tau)\right|^{2}=2 \ln (y-C \beta)
$$

Up to now we have followed the treatment just in the $\left(z_{i}, \tau\right)$-sector (cf. our discussion in the subsection on the corresponding restricted preconsideration). Now we have to properly enhance these results by going to the consideration of the full $\left(z_{i}, \tau, \mathcal{T}_{k}\right)$-sector. 


\section{Including the $\mathcal{T}_{i}$-sector}

Up to now we have expressed the $z_{i}$ as functions of $\tau$ from $D_{i} W_{\text {flux }}=0$. According to the discussion in sect. 5.2 we want actually to obtain $z_{i}\left(\tau, \mathcal{T}_{i}\right)$ from $D_{i}\left(W_{\text {flux }}+W^{(\mathcal{T})}\right)=0$. For this we will 1) give the explicit expressions $\left.z_{i}(\tau), 2\right)$ generalize our flux-example to include a constant flux and give there the $\left.z_{i}(\tau), 3\right)$ interpret formally the additional term $W^{(\mathcal{T})}$, which is constant in $\tau$, as such an additional flux term and 4) give thereby the full $z_{i}\left(\tau, \mathcal{T}_{i}\right)$.

1) Note first that $\operatorname{Re} W_{\text {flux }}=0$ from (6.31) gives

$$
x_{1}=\frac{g}{A_{1}-3 B_{1}}\left(\frac{1}{2} X^{2}+\frac{1}{2} \beta^{2}-\left(\frac{3}{2}+\frac{B_{1}}{g}\right) X-\frac{17}{4}\right)
$$

Therefore the two variables $Z=3 z_{1}+z_{2}=X+i Y$ and $z_{1}=x_{1}+i y_{1}$ are completely determined as functions of $\tau$ and $y=y_{2}$ (and therefore as functions of $\tau$ alone): for $X$ and $Y$ this was given above, and for $z_{1}$ it is, together with $y_{1}=\left(Y-y_{2}\right) / 3$, given here.

2) Now let us generalize this set-up by including an additional constant (in the $z_{i}$ ) contribution in $W_{f l u x}$. For this we start with the enhanced flux vector

$$
\left(e_{R}^{a} \mid m_{R}^{a}\right)=(0, g, 0 \mid-e,-a,-b) \quad, \quad\left(e_{N S}^{a} \mid m_{N S}^{a}\right)=\left(0,0,0 \mid e^{\prime}, c, d\right)
$$

which gives (with $E=e+e^{\prime} \tau=E_{1}+i E_{2}$ ) the flux-superpotential $W_{\text {flux }}^{\prime}=W_{\text {flux }}+E$

$$
W_{f l u x}^{\prime}=g F_{1}+A z_{1}+B z_{2}+E
$$

In this generalized situation (with $p \tilde{E}=E$ ) the elimination of $y$ reads now (the expressions (6.34) for $X$ and $Y$, coming from (6.33), have still just $g$ and $C$ and remain the same)

$$
y^{3}-3\left(\beta C+\tilde{E}_{2}\right) y^{2}+2\left(\beta^{3}+9 i \xi\right)=0
$$

Formally one could even absorb the $\tilde{E}_{2}=: \tilde{e} \tau_{2}=p e^{\prime} \tau_{2}$ in a $\tilde{C}$ built with an $\tilde{g}:=g\left(1+\frac{2 e^{\prime}}{3 d}\right)$, i.e., $\beta C+\tilde{E}_{2}=\beta \tilde{C}$ (not to be used in (6.34)). Finally one gets the shift $x_{1}^{\prime}=x_{1}-\frac{E_{1}}{a-3 b}$.

3) Now the elimination condition for $z_{i}=z_{i}\left(\tau, \mathcal{T}_{i}\right)$

$$
\partial_{i} W_{\text {flux }}+K_{i}\left(W_{\text {flux }}+W^{(\mathcal{T})}\right)=0
$$

can formally be interpreted as the elimination condition $\partial_{i} W_{\text {flux }}^{\prime}+K_{i} W_{\text {flux }}^{\prime}=0$ for $z_{i}=z_{i}(\tau)$ with respect to an enhanced flux-superpotential $W_{\text {flux }}^{\prime}=W_{\text {flux }}+E$ where the latter will be interpreted formally as $W^{(\mathcal{T})}$.

4) Alltogether we get thereby the searched for expressions $z_{i}\left(\tau, \mathcal{T}_{i}\right)$ as the $z_{i}^{\prime}(\tau)$ in the previous set-up, generalized by putting formally $E:=W^{(\mathcal{T})}$. 


\subsubsection{The SUSY conditions for the Kähler moduli $\mathcal{T}_{i}$ and the dilaton $\tau$}

Let us now apply the previous considerations concerning $G_{\text {eff }}$ in (5.15) to our twoparameter model. In the end we will discuss stable minima for $V$ in (5.1). One finds, for $^{9} y$ in (6.48), with the help of (6.41) that (up to an additive constant)

$$
K_{\text {eff }}\left(\tau, \mathcal{T}_{i}\right)=K\left(z_{i}\left(\tau, \mathcal{T}_{i}\right)\right)=K\left(z_{i}^{\prime}(\tau)\right)=-\ln y^{2}-\ln (y-\beta \tilde{C})
$$

Similarly one has with $E=W^{(\mathcal{T})}, E=p \tilde{E}$ and $\beta C+\tilde{E}_{2}=\beta \tilde{C}$ where $\tilde{C}$ is a function not only of $\tau_{1}$, as in $(6.28)$, but also of the $\mathcal{T}_{i}\left(\right.$ with $W^{(\mathcal{T})}\left(\mathcal{T}_{i}\right)=\sum C_{k} e^{-a_{k} \mathcal{T}_{k}}$ )

$$
\tilde{C}=\frac{a+c \tau_{1}+\frac{9}{2} g+\frac{3 g}{d \tau_{2}} \operatorname{Im} W^{(\mathcal{T})}\left(\mathcal{T}_{i}\right)}{a-3 b}
$$

(for simplicity we will assume $C_{i} \in \mathbf{R}$ ) the following expression

$$
\begin{aligned}
W_{\text {eff }}\left(\tau, \mathcal{T}_{i}\right)=W_{\text {flux }}\left(z_{i}\left(\tau, \mathcal{T}_{i}\right), \tau\right) & =W_{\text {flux }}\left(z_{i}^{\prime}(\tau)\right)=i\left[p(\beta C-y)+\operatorname{Im} W^{(\mathcal{T})}\right] \\
& =i p(\beta \tilde{C}-y)
\end{aligned}
$$

Therefore we find alltogether for the $G_{\text {eff }}$ in (5.15) (up to an additive constant)

$$
\begin{aligned}
G_{\text {eff }}= & -\ln (\tau-\bar{\tau})+K_{\text {eff }}\left(\tau, \mathcal{T}_{i}\right)+\ln \left|W_{\text {eff }}\left(\tau, \mathcal{T}_{i}\right)+W^{(\mathcal{T})}\left(\mathcal{T}_{i}\right)\right|^{2}+K\left(\mathcal{T}_{i}\right) \\
= & -\ln (\tau-\bar{\tau})-2 \ln y-\ln (y-\beta \tilde{C})+\ln \left|i p(\beta \tilde{C}-y)+\sum_{i=1}^{2} C_{i} e^{-a_{i} \mathcal{T}_{i}}\right|^{2} \\
& -2 \ln \left(\left(\mathcal{T}_{2}+\overline{\mathcal{T}}_{2}\right)^{3 / 2}-\left(\mathcal{T}_{1}+\overline{\mathcal{T}}_{1}\right)^{3 / 2}\right)
\end{aligned}
$$

from which one gets the three $S U S Y$ conditions $G_{i}=0$. When searching for solutions one has to satisfy also $L=N_{\text {flux }}=c g / 2 \leq e\left(X_{4}\right) / 24=273$ and 129 for $a_{2}=1 / 12$ and $1 / 30$, respectively. (When using $W^{(\mathcal{T})}$ numerically one has to make the shift $a_{i} \rightarrow 2 \pi a_{i}$.)

Using $\beta=\frac{d}{g} \tau_{2}$, and $\beta \tilde{C}=\beta C+\frac{1}{p} \operatorname{Im} W^{(T)}$ one has

$$
\begin{array}{ccc}
\beta_{\tau}=\frac{d}{2 i g} \quad, \quad(\beta \tilde{C})_{\tau}=\beta_{\tau} C+\beta \frac{c / 2}{a-3 b} \\
\beta_{\mathcal{T}_{i}}=0 \quad, \quad(\beta \tilde{C})_{\mathcal{T}_{k}}=\frac{i}{2 p} a_{k} C_{k} e^{-a_{k} \mathcal{T}_{k}}
\end{array}
$$

From (6.48) one finds $\left(l\right.$ is $\tau$ or $\left.\mathcal{T}_{i}\right)$

$$
y_{l}(y-2 \beta \tilde{C})=(\beta \tilde{C})_{l} y-2 \frac{\beta_{l} \beta^{2}}{y}
$$

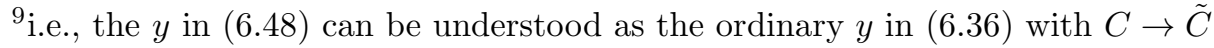


Evaluating the SUSY conditions

For evaluation of the SUSY conditions we use the following abbreviations

$$
\begin{gathered}
\mathcal{B}=\beta \tilde{C}-y \quad, \quad s_{k}=-(-1)^{k} \frac{3}{2} \frac{t_{k}^{1 / 2}}{t_{2}^{3 / 2}-t_{1}^{3 / 2}} \quad, \quad W^{T}=\sum_{k=1}^{2} W^{k}=\sum_{k=1}^{2} C_{k} e^{-a_{k}\left(t_{k}+i \theta_{k}\right)}(6.58) \\
\Gamma=p \mathcal{B} \frac{p \mathcal{B}+\operatorname{Im} W^{T}}{\left(p \mathcal{B}+\operatorname{Im} W^{T}\right)^{2}+\left(\operatorname{Re} W^{T}\right)^{2}} \quad, \quad \Gamma^{\prime}=\frac{-i\left(p \mathcal{B}+\operatorname{Im} W^{T}\right)+\operatorname{Re} W^{T}}{\left(p \mathcal{B}+\operatorname{Im} W^{T}\right)^{2}+\left(\operatorname{Re} W^{T}\right)^{2}}(6.59)
\end{gathered}
$$

$\left(\mathcal{B}, s_{k}, \Gamma\right.$ are real quantities). One gets for the SUSY conditions

$$
\begin{aligned}
& G_{k}=-2 \frac{y_{k}}{y}+(2 \Gamma-1) \frac{\mathcal{B}_{k}}{\mathcal{B}}-a_{k} W^{k} \Gamma^{\prime}+s_{k}=0 \\
& G_{\tau}=-2 \frac{y_{\tau}}{y}+(2 \Gamma-1) \frac{\mathcal{B}_{\tau}}{\mathcal{B}}+\frac{i}{2 \tau_{2}}=0
\end{aligned}
$$

Note that $y \neq 0$ or else one would have $\beta^{3}+9 i \xi=0$ by the cubic equation and $K\left(z_{i}\right)=$ $\ln \left(Y^{3}-y^{3}+9 i \xi\right)=\ln \left(\beta^{3}-y^{3}+9 i \xi\right)$ would degenerate. Note furthermore that $y-2 \beta \tilde{C} \neq 0$ or else (6.57) would give $\left(\beta \frac{c / 2}{a-3 b}+\frac{d}{2 i g} C\right) y^{2}=2 \frac{d}{2 i g} \beta^{2}$, i.e. $c=0=d$ from the real part, violating our assumption. Applying (6.57) to $l=k$ likewise gives the immediate contradiction $C_{k}=0$.

For the following it is useful to collect some expressions (subscript $k$ indicates $\partial_{\mathcal{T}_{k}}$ )

$$
\begin{gathered}
(\beta \tilde{C})_{k}=\frac{i}{2 p} a_{k} W^{k} \\
y_{k}=(\beta \tilde{C})_{k} \frac{y}{y-2 \beta \tilde{C}} \quad, \quad \mathcal{B}_{k}=-(\beta \tilde{C})_{k} \frac{2 \beta \tilde{C}}{y-2 \beta \tilde{C}}
\end{gathered}
$$

As the splitting in real and complex parts will be crucial below note that here the fractions are real so that the real and imaginary parts of $y_{k}$ and $\mathcal{B}_{k}$ go with the corresponding real and imaginary parts of $(\beta \tilde{C})_{k}$ (times the fraction).

Similarly one gets in the $\tau$-sector

$$
\begin{gathered}
(\beta \tilde{C})_{\tau}=\beta \frac{c / 2}{3 p}-i \frac{d}{2 g} C \\
\operatorname{Re} y_{\tau}=\operatorname{Re}(\beta \tilde{C})_{\tau} \frac{y}{y-2 \beta \tilde{C}} \quad, \quad \operatorname{Re} \mathcal{B}_{\tau}=-\operatorname{Re}(\beta \tilde{C})_{\tau} \frac{2 \beta \tilde{C}}{y-2 \beta \tilde{C}} \\
\operatorname{Im} y_{\tau}=-\frac{d}{2 g} \frac{C y^{2}-2 \beta^{2}}{y(y-2 \beta \tilde{C})} \quad, \quad \operatorname{Im} \mathcal{B}_{\tau}=-\frac{d}{2 g} \frac{2 \beta^{2}-2 \beta \tilde{C} C y}{y(y-2 \beta \tilde{C})}
\end{gathered}
$$


Now let us consider the real and imaginary parts of $G_{k}=0$ and $G_{\tau}=0$

$$
\begin{aligned}
-2 \frac{\operatorname{Re} y_{k}}{y}+(2 \Gamma-1) \frac{\operatorname{Re} \mathcal{B}_{k}}{\mathcal{B}}-a_{k} \frac{\operatorname{Re}\left[W^{k}(-i p \mathcal{B}+\bar{W})\right]}{|i p \mathcal{B}+W|^{2}} & =-s_{k} \\
-2 \frac{\operatorname{Im} y_{k}}{y}+(2 \Gamma-1) \frac{\operatorname{Im} \mathcal{B}_{k}}{\mathcal{B}}-a_{k} \frac{\operatorname{Im}\left[W^{k}(-i p \mathcal{B}+\bar{W})\right]}{|i p \mathcal{B}+W|^{2}} & =0 \\
-2 \frac{\operatorname{Re} y_{\tau}}{y}+(2 \Gamma-1) \frac{\operatorname{Re} \mathcal{B}_{\tau}}{\mathcal{B}} & =0 \\
-2 \frac{\operatorname{Im} y_{\tau}}{y}+(2 \Gamma-1) \frac{\operatorname{Im} \mathcal{B}_{\tau}}{\mathcal{B}} & =-\frac{1}{2 \tau_{2}}
\end{aligned}
$$

(here $W:=W^{T}$ ). Let us draw some consequences (we had $C_{i} \in \mathbf{R}$ for simplicity)

$$
\begin{array}{rlrl}
2 \mathcal{B} & =-2(2 \Gamma-1) \beta \tilde{C} & (\Longrightarrow y=2 \Gamma \beta \tilde{C}) \\
R e W^{k}=0 \Longrightarrow \theta_{k}= \pm \frac{\pi}{a_{k}} \text { and } \operatorname{Re} W=0 & \left(\Longrightarrow \Gamma=\frac{p \mathcal{B}}{p \mathcal{B}+\operatorname{Im} W}\right) \\
\frac{s_{k}}{a_{k}}=\frac{I m W^{k}}{p \mathcal{B}+\operatorname{Im} W}=\frac{ \pm C_{k} e^{-a_{k} t_{k}}}{p \mathcal{B}+\sum \pm C_{j} e^{-a_{j} t_{j}}} \quad\left(\Longrightarrow \sum \frac{s_{k}}{a_{k}}=\frac{\operatorname{Im} W}{p \mathcal{B}+\operatorname{Im} W}\right) \\
\tilde{C} y^{2}=-2 \beta^{2} & \left(\stackrel{(6.36)}{\Longrightarrow} y^{3}+2\left(4 \beta^{3}+9 i \xi\right)=0\right)
\end{array}
$$

\section{Proof of $(6.71)-(6.74)$}

(6.69) implies (6.71). This makes the first two terms in (6.68) cancel

$$
0=\frac{a_{k}}{2 p} \operatorname{Re} W^{k}\left(-2 \frac{1}{y-2 \beta \tilde{C}}+(2 \Gamma-1) \frac{-2 \beta \tilde{C}}{\mathcal{B}(y-2 \beta \tilde{C})}\right)-a_{k} \frac{\operatorname{Im}\left[W^{k}(-i p \mathcal{B}+\bar{W})\right]}{|i p \mathcal{B}+W|^{2}}
$$

which proves (6.72). With (6.63) and (6.71) one gets that in (6.67) the first two terms cancel so that one gets the following relation implying (6.73) using $\operatorname{Re} W=0$

$$
\frac{s_{k}}{a_{k}}=\frac{R e\left[W^{k}(-i p \mathcal{B}+\bar{W})\right]}{|i p \mathcal{B}+W|^{2}}
$$

Rewriting (6.70) with (6.71) and multiplying by $-2 \tau_{2} y^{2} \tilde{C}$ gives (6.74).

By (6.74) one gets $y$ as function of $\beta$ (i.e. of $\tau_{2}$ ); similarly for $\tilde{C}, \mathcal{B}$ and $\Gamma$ (let $\zeta:=9 i \xi$ )

$$
\begin{aligned}
y & =\left[-2\left(4 \beta^{3}+\zeta\right)\right]^{1 / 3} \\
\tilde{C} & =-\frac{2 \beta^{2}}{y^{2}}=-\frac{2 \beta^{2}}{\left[-2\left(4 \beta^{3}+\zeta\right)\right]^{2 / 3}} \\
\mathcal{B} & =\beta \tilde{C}-y=\left[-2\left(4 \beta^{3}+\zeta\right)\right]^{1 / 3}\left(\frac{\beta^{3}}{\left(4 \beta^{3}+\zeta\right)}-1\right) \\
\Gamma & =\frac{y}{2 \beta \tilde{C}}=\frac{4 \beta^{3}+\zeta}{2 \beta^{3}}
\end{aligned}
$$

So there remain three equations for the unknowns $t_{1}, t_{2}, \tau_{2}$. When one has obtained $t_{1}, t_{2}, \tau_{2}$ one will get $\tau_{1}$ from the definition (6.51) of $\tilde{C}$. 
One remains with the three equations (for $(6.82)$ note $\sum \frac{s_{k}}{a_{k}}=\frac{\operatorname{Im} W}{p \mathcal{B}+\operatorname{Im} W}=1-\Gamma$ )

$$
\begin{aligned}
\frac{s_{k}}{a_{k}} & =\frac{\operatorname{Im} W^{(k)}}{p \mathcal{B}+\operatorname{Im} W} \\
\mathcal{S}:=\sum \frac{s_{k}}{a_{k}} & =-\frac{2 \beta^{3}+\zeta}{2 \beta^{3}}
\end{aligned}
$$

Now let us decouple the $t_{i}$ sector from the $\tau_{2}$ sector. (6.81) gives first

$$
\begin{aligned}
-\sqrt{\frac{t_{2}}{t_{1}}}=\frac{s_{2}}{s_{1}} & =\frac{a_{2}}{a_{1}} \frac{ \pm_{2} C_{2} e^{-a_{2} t_{2}}}{ \pm_{1} C_{1} e^{-a_{1} t_{1}}} \\
\mathcal{S} & \left.=\frac{\operatorname{Im} W}{p \mathcal{B}+\operatorname{Im} W} \quad \text { (with } \mathcal{B}=\sqrt[3]{-2 \zeta \frac{\mathcal{S}-1}{\mathcal{S}+1}} \frac{\frac{1}{2}-\mathcal{S}}{\mathcal{S}-1}\right)
\end{aligned}
$$

For (6.84) note (6.79) and that (6.82) gives $\beta^{3}=-\frac{1}{2} \frac{\zeta}{1+\mathcal{S}}$; the latter relation will give $\tau_{2}$ once we have solved the two equations (6.83) and (6.84) for the $t_{i}$.

These equation can also be written as (note $\mathcal{S}=\sum \frac{s_{k}}{a_{k}}$ and $\operatorname{Im} W=\sum \operatorname{Im} W^{(k)}$ )

$$
\begin{aligned}
& \frac{s_{2} / a_{2}}{s_{1} / a_{1}}=\frac{\operatorname{Im} W^{(2)}}{\operatorname{Im} W^{(1)}} \\
& \operatorname{Im} W=p \sqrt[3]{-2 \zeta} \frac{\mathcal{S}\left(\mathcal{S}-\frac{1}{2}\right)}{\mathcal{S}^{2}-1}
\end{aligned}
$$

Obviously these equations could possibly have the trivial solution that the common proportionality factor in (6.85) is -1 (if that value can be realised); this then means however that $\mathcal{S}=0=\operatorname{Im} W$ and $\Gamma=1$, so that $y-2 \beta \tilde{C}=0$, according to (6.80), contrary to hypothesis (it would imply $c=0=d$ ).

Let us recall finally also the explicit expressions for the independent variables $t_{k}$

$$
s_{k}=(-1)^{k+1} \frac{3}{2} \frac{t_{k}^{1 / 2}}{t_{2}^{3 / 2}-t_{1}^{3 / 2}} \quad, \quad t_{k}=-\frac{3}{2} \frac{s_{k}^{2}}{s_{1}^{3}+s_{2}^{3}} \quad ; \quad \operatorname{Im} W^{(k)}= \pm_{k} C_{k} e^{-a_{k} t_{k}}
$$

The solution to $(6.85),(6.86)$ is (we take $\pm_{k}=+$, it can be absorbed in $C_{k}$ anyway)

$$
a_{k} t_{k}=\frac{a_{k}^{3}}{a_{2}^{3}-a_{1}^{3}} L \Longrightarrow \frac{s_{k}}{a_{k}}=(-1)^{k+1} \frac{3}{2 L}, \quad \operatorname{Im} W^{(k)}=(-1)^{k+1} \frac{C_{1}^{\frac{a_{2}^{3}}{a_{2}^{3}-a_{1}^{3}}}}{\left(-C_{2}\right)^{\frac{a_{1}^{3}}{a_{2}^{3}-a_{1}^{3}}}}
$$

(where $L:=\ln \left(-C_{2} / C_{1}\right)$ ) such that (note the independence of $p$ ) one has only the mentioned trivial solution $\mathcal{S}=0=\operatorname{Im} W$. Thus there are no SUSY solutions.

Note that the conclusion would not change if one includes more generally a proper flux $E=e+e^{\prime} \tau$ (not just to mimic formally $\left.W^{(T)}\right)$ : one gets then for $\beta \tilde{\mathcal{C}}:=\beta C+E+W^{(T)}$ that $(\beta \tilde{\mathcal{C}})_{\tau}=\beta_{\tau} C_{E}+\beta \frac{c / 2}{a-3 b}$ with $C_{E}=C+\frac{g}{d} e^{\prime}$ leaving (6.74) intact. 


\subsection{The case III: $g+\frac{1}{3} h=0$}

For the flux components (again sticking to the case $c=3 d$ )

$$
\left(e_{R}^{a} \mid m_{R}^{a}\right)=(0, g, h \mid 0,-a,-b) \quad, \quad\left(e_{N S}^{a} \mid m_{N S}^{a}\right)=(0,0,0 \mid 0, c, d)
$$

one gets the flux superpotential $W_{\text {flux }}=g F_{1}+h F_{2}+A z_{1}+B z_{2}$ or

$$
W_{\text {flux }}=g\left(-\frac{1}{2} Z^{2}+\frac{3}{2} Z+\frac{17}{4}\right)+\frac{h}{3}\left(-\frac{1}{2}\left(Z^{2}-z_{2}^{2}\right)+\frac{3}{2}\left(Z-z_{2}\right)+\frac{18}{4}\right)+\frac{A}{3} Z-p z_{2}
$$

One gets as elimination equations $\frac{1}{3} D_{z_{1}} W=0$ and $D_{z_{2}} W=0$

$$
\begin{aligned}
g\left(-Z+\frac{3}{2}\right)+\frac{h}{3}\left(-Z+\frac{3}{2}\right)+\frac{A}{3} & =\frac{3}{2 i} \frac{Y^{2}}{Y^{3}-y^{3}+9 i \xi} W_{\text {flux }} \\
g\left(-Z+\frac{3}{2}\right)+\frac{h}{3}\left(-Z+z_{2}\right)+B & =\frac{3}{2 i} \frac{Y^{2}-y^{2}}{Y^{3}-y^{3}+9 i \xi} W_{f l u x}
\end{aligned}
$$

and the compatibility equation reads

$$
\left[\frac{h}{3}\left(\frac{3}{2}-z_{2}\right)+p\right] Y^{2}=\left[\left(g+\frac{h}{3}\right)\left(-Z+\frac{3}{2}\right)+\frac{A}{3}\right] y^{2}
$$

Now let us introduce again a linear binding between $g$ and $h$. Whereas previously we had set $h=0$ we put now $g+\frac{h}{3}=0$. This gives

$$
\left[\frac{h}{3}\left(\frac{3}{2}-z_{2}\right)+p\right] Y^{2}=\frac{A}{3} y^{2} \stackrel{y=Y^{2} / \beta}{\Longrightarrow} g\left(z_{2}-\frac{3}{2}\right)+p=\frac{A}{3}\left(\frac{Y}{\beta}\right)^{2}
$$

(taking the imaginary part gives the indicated relation for $y$ ). One gets then for $W_{\text {flux }}$

$$
W_{\text {flux }}=g\left(-\frac{1}{2} z_{2}^{2}+\left(\frac{3}{2}-\frac{p}{g}\right) z_{2}-\frac{1}{4}\right)+\frac{A}{3} Z
$$

Equating with (6.90) gives

$$
\frac{A}{3} \frac{2 i}{3} \frac{Y^{3}-\beta^{-3} Y^{6}+9 i \xi}{Y^{2}}=g\left(\frac{1}{32}-\frac{3 p}{2 g}+\frac{p^{2}}{2 g^{2}}\right)-\frac{1}{2 g}\left[g\left(z_{2}-\frac{3}{2}\right)+p\right]^{2}+\frac{A}{3} Z
$$

One gets thereby as effective 'superpotential'

$$
W_{e f f}=\frac{A}{3} \frac{2 i}{3} \frac{Y^{3}-\beta^{-3} Y^{6}+9 i \xi}{Y^{2}}
$$

where $Y$ is determined by (gotten from taking $A_{2} \operatorname{Re}-A_{1} \operatorname{Im}$ of (6.95) to eliminate $X$ )

$$
\beta^{-3} Y^{6}+2 Y^{3}+C Y^{2}-4 \cdot 9 i \xi=0
$$

One can not tune this to become a quadric in $Y^{3}$ as the demand for vanishing of

$$
C=-\frac{27}{16} \frac{\beta}{A_{1}^{2}+A_{2}^{2}}\left(g^{2}-48 p g+16 p^{2}\right)
$$

leads to the condition $g=4(6 \pm \sqrt{35}) p$, which can not be solved in integral fluxes. 
However including again the fluxes $m_{R}^{0}=-e, m_{N S}^{0}=e^{\prime}$ and the ensuing term $E=$ $e+e^{\prime} \tau$ in $W_{\text {flux }}$ leads effectively to the following coefficient in the sextic

$$
\begin{aligned}
\tilde{C} & =-\frac{27}{16} \frac{\beta}{A_{1}^{2}+A_{2}^{2}}\left[\left(g^{2}-48 p g+16 p^{2}\right)+\frac{32 g}{A_{2}}\left(A_{2} E_{1}-A_{1} E_{2}\right)\right] \\
& =-\frac{27}{16} \frac{\beta}{A_{1}^{2}+A_{2}^{2}}\left(g^{2}-48 p\left[1-\frac{2\left(e-\frac{a}{c} e^{\prime}\right)}{3 p}\right] g+16 p^{2}\right)
\end{aligned}
$$

This is easily solved in special cases, such as $e-\frac{a}{c} e^{\prime}=2 p$ giving $g=-4 p$ leading to the sextic being just quadratic in $Y^{3}$. However as the $E$-sector is used by us to include the $W^{(\mathcal{T})}$-dependence formally, giving the $z_{i}\left(\tau, \mathcal{T}_{i}\right)$, any special tuning of it can not be kept under the further procedure, such that one will have still to face the more general sextic. When the $E$-sector is used for this purpose (to just mimic the $W^{(\mathcal{T})}$ ) one has to use just the expression (6.99). So one has now to work with the sextic with $C$ replaced by $\tilde{C}$.

Now $\beta=\frac{d}{g} \tau_{2}$, gives $\beta_{\tau}=\frac{d}{2 i g}, \beta_{\mathcal{T}_{k}}=0$ and (6.99) gives $\tilde{C}_{\mathcal{T}_{k}}=i \frac{9}{2 A} a_{k} W^{k}$ and a more complicated expression for $\tilde{C}_{\tau}$. From (6.97) with $C \rightarrow \tilde{C}$ one finds $Y_{l}\left(\beta^{-3} Y^{4}+Y+\frac{\tilde{C}}{3}\right)=$ $\frac{1}{2}\left(\beta^{-4} \beta_{l} Y^{4}-\frac{\tilde{C}_{l}}{3}\right) Y$ (here $l$ is $\tau$ or $\left.\mathcal{T}_{i}\right)$. With the notation $\mathcal{B}:=-\beta^{-3} Y^{4}+2 Y+\frac{\tilde{C}}{3}$ one gets for the effective Kahler potential (up to additive constants) and superpotential

$$
\begin{aligned}
& K_{\text {eff }}(\tau)=-\ln \left(Y^{3}-y^{3}+9 i \xi\right)=-2 \ln Y-\ln \mathcal{B} \\
& W_{\text {eff }}(\tau)=-i \mathcal{A} \mathcal{B} \quad\left(\mathcal{A}:=\frac{A}{6}\right)
\end{aligned}
$$

One gets as effective $G$-function and SUSY conditions (note the marked difference in this case that now $A \in \mathbf{C}$ is $\tau$-dependent whereas earlier $p \in \mathbf{R}$ was a constant)

$$
\begin{aligned}
G & =-\ln (\tau-\bar{\tau})-2 \ln Y-\ln \mathcal{B}+\ln \left|i \mathcal{A} \mathcal{B}+W^{(\mathcal{T})}\right|^{2}+K\left(\mathcal{T}_{k}\right) \\
G_{k} & =-2 \frac{y_{k}}{y}+(2 \Gamma-1) \frac{\mathcal{B}_{k}}{\mathcal{B}}-a_{k} W^{k} \Gamma^{\prime}+s_{k}=0 \\
G_{\tau} & =-2 \frac{y_{\tau}}{y}+(2 \Gamma-1) \frac{\mathcal{B}_{\tau}}{\mathcal{B}}+i \frac{c}{6} \mathcal{B} \Gamma^{\prime}+\frac{i}{2 \tau_{2}}=0
\end{aligned}
$$

where we use the abbreviations

$\Gamma=\mathcal{B} \frac{\mathcal{A}_{1}\left(\operatorname{Im} W^{(T)}+\mathcal{A}_{1} \mathcal{B}\right)-\mathcal{A}_{2}\left(\operatorname{Re} W^{(T)}-\mathcal{A}_{2} \mathcal{B}\right)}{\left|i \mathcal{A B}+W^{(T)}\right|^{2}}, \Gamma^{\prime}=\frac{-i\left(\operatorname{Im} W^{(T)}+\mathcal{A}_{1} \mathcal{B}\right)+\left(\operatorname{Re} W^{(T)}-\mathcal{A}_{2} \mathcal{B}\right)}{\left|i \mathcal{A B}+W^{(T)}\right|^{2}}$

One can now start to proceed as in the previous examples. We will not write the ensuing equations here, as they become not especially illuminating. However, by now the general scheme should be rather clear. Proceeding in the manner described, the interested reader should be able to use this method to work out further cases. Note that each case will be of a somewhat different nature (cf. the remark here after (6.102)), and furthermore although for more general flux combinations the existence of SUSY vacua becomes more likely, on the other hand the explicit determination of $W_{\text {eff }}$ also becomes more involved. 


\section{Discussion and Outlook}

One of the most often studied scenarios of moduli stabilization is the KKLT set-up. This leads to supersymmetric AdS vacua with all moduli stabilized. Eventually this is uplifted to a SUSY-breaking dS vacuum. As the uplift process is not easily treated in a controlled framework other approaches to get moduli stabilization and dS vacua are of course of the highest interest [18], [19].

In this paper we stick just to the moduli stabilization aspect of the KKLT scenario. The common procedure employed in the KKLT framework is to use first [1] to fix the complex structure moduli $z_{i}$ and the dilaton $\tau$ from a flux superpotential $W_{\text {flux }}$ and then, afterwards, to use non-perturbative effects to stabilize the Kähler moduli $\mathcal{T}_{k}$. The underlying assumptions of the decoupling in this two-step procedure have been questioned [7] (cf. also [20]). This led to a procedure where only the $z_{i}$ are integrated out what leads to an effective superpotential $W_{\text {eff }}(\tau)$ (in [7] conditions for a valid uplift were also considered). As this $W_{\text {eff }}(\tau)$ is a non-holomorphic quantity, as already pointed out in [8], the whole procedure actually has to be rephrased with the aid of the $G$-function of supergravity. When the integrating out-process is incorporated appropriately one actually gets not only a $W_{\text {eff }}(\tau)$ but a $W_{\text {eff }}\left(\tau, \mathcal{T}_{k}\right)$; it then contributes via the $\ln \left|W_{\text {eff }}\left(\tau, \mathcal{T}_{k}\right)+W^{(\mathcal{T})}\left(\mathcal{T}_{k}\right)\right|^{2}$ term in the effective $G$-function.

This program, which was described in [7] and [8] in a generic effective supergravity framework, is carried out here for one of the most intensely studied Calabi-Yau related models, the orientifold model of $\mathbf{P}_{11169}[18]$, which was also studied in the original KKLT framework [9]. Even more, this geometry was also the background to study further questions, such as inclusion of $\alpha^{\prime 3}$-corrections [11], [12] or inflation [10].

The new approach is investigated here for this geometry in various flux combinations. More precisely, the determination of $W_{e f f}$ is illustrated mainly for the case

$$
\left(e_{R}^{a} \mid m_{R}^{a}\right)=(0, g, 0 \mid 0,-a,-b) \quad, \quad\left(e_{N S}^{a} \mid m_{N S}^{a}\right)=(0,0,0 \mid 0, c, d)
$$

One gets for $g \neq 0$ as effective superpotential (the case II above)

$$
W_{\text {eff }}\left(\tau, \mathcal{T}_{k}\right)=i \frac{a-3 b}{3}\left(\frac{d}{g} \tau_{2} \frac{a+c \tau_{1}+\frac{9}{2} g+\frac{3 g}{d \tau_{2}} \operatorname{Im} W^{(\mathcal{T})}\left(\mathcal{T}_{i}\right)}{a-3 b}-y\right)
$$

Here $y$ is given as function of $\tau$ and $\mathcal{T}_{k}$ by the Cardano formula for the cubic

$$
0=y^{3}-\left[3 \frac{d}{g} \tau_{2} \frac{a+c \tau_{1}+\frac{9}{2} g+\frac{3 g}{d \tau_{2}} \operatorname{Im} W^{(\mathcal{T})}\left(\mathcal{T}_{i}\right)}{a-3 b}\right] y^{2}+2\left(\left(\frac{d}{g} \tau_{2}\right)^{3}+9 i \xi\right)
$$

Here $W^{(\mathcal{T})}$ denotes $\sum_{k=1}^{2} C_{k} e^{-a_{k} \mathcal{T}_{k}}$. 
Above we did assume that $g \neq 0$. In the case $g=0$ one gets (case I above)

$$
W_{\text {eff }}\left(\tau, \mathcal{T}_{k}\right)=i\left(d \tau+\frac{a}{3}\right)\left(Y+3 \frac{\left(a+c \tau_{1}\right) \operatorname{Im} W^{(\mathcal{T})}-c \tau_{2} \operatorname{Re} W^{(\mathcal{T})}}{|a+c \tau|^{2}}\right)
$$

Again, here $Y$ is given as function of $\tau$ and $\mathcal{T}_{k}$ by the Cardano formula for the cubic

$$
0=Y^{3}+9\left[\frac{\left(a+c \tau_{1}\right) \operatorname{Im} W^{(\mathcal{T})}-c \tau_{2} \operatorname{Re} W^{(\mathcal{T})}}{|a+c \tau|^{2}}\right] Y^{2}-18 i \xi
$$

In these cases one can resolve analytically the remaining supersymmetry conditions on $\tau$ and the $\mathcal{T}_{k}$. As the case $g=0$ is somewhat degenerate $\left(N_{\text {flux }}=0\right)$ we give the treatment fully only for our main case $g \neq 0$. There one finds that no supersymmetric solutions to the ensuing supersymmetry conditions for $\tau$ and the $\mathcal{T}_{k}$ exist.

There is a third, more complicated case with a non-zero $e_{R}^{2}$ turned on (the case III above), where we also give the effective superpotential and pave the way for the treatment of the supersymmetry conditions. As in the present paper our aim was just to elucidate the concrete improved KKLT procedure in the spirit of [7] and [8], we are content here to illustrate this new set-up with a number of non-trivial examples where actually all the steps can be carried out in closed analytical form (this program is surely completely worked out at least for the main case $g \neq 0$, i.e. case II).

The worked out examples should make it sufficiently clear how the preceding considerations could be generalised further. One next step would be to 'solve' the $\mathbf{P}_{11169}[18]$ model in the sense of allowing (more) general flux combinations. It would be especially interesting to turn on the flux $e_{R}^{0}$ which leads to a contribution $e_{R}^{0} F_{0}$ in $W_{\text {flux }}$ which would be even of cubic degree in the $z_{i}$ and so will lead to a more complicated dependence in $W_{\text {eff }}$. As the treatment [9] along the lines of the original KKLT set-up contains this flux this would open up the possibility for a direct comparison. Of course alternatively one can study the KKLT procedure also in one of the simpler examples by demanding $D_{\tau} W_{\text {flux }}=0$ in addition to $D_{z_{i}} W_{\text {flux }}=0$. We indicated this in (6.13) and a similar analysis is easily given for $g \neq 0$. Another interesting avenue to explore would be to combine the appropriate modification of the KKLT procedure outlined here with further questions such as the inclusion of $\alpha^{\prime 3}$-corrections [11], [12] or inflation [10]. Furthermore one could carry out the program described here for other examples of low parameter Calabi-Yau (orientifold) models. Finally one could, following [7], work out the appropriate criterion for a successful uplift to dS space in connection with the question of stability, i.e. one could study the nature of the supersymmetric points with respect to the stronger dS stability criterion. Touching thereby the realm of non-supersymmetric solutions one could study numerically their existence already for the potential arising here (without uplift).

We would like to thank S. Theisen for participation at some stages of this work. 


\section{A SUSY conditions in case I}

Let us consider the real and imaginary parts of $G_{k}=0$ and $G_{\tau}=0\left(\right.$ with $\left.W:=W^{(\mathcal{T})}\right)$

$$
\begin{aligned}
-2 \frac{\operatorname{Re} Y_{k}}{Y}+(2 \Gamma-1) \frac{\operatorname{Re} \mathcal{B}_{k}}{\mathcal{B}}-a_{k} \frac{\operatorname{Re}\left[W^{k}(-i \overline{\mathcal{A}} \mathcal{B}+\bar{W})\right]}{|i \mathcal{A B}+W|^{2}} & =-s_{k} \\
-2 \frac{\operatorname{Im} Y_{k}}{Y}+(2 \Gamma-1) \frac{\operatorname{Im} \mathcal{B}_{k}}{\mathcal{B}}-a_{k} \frac{\operatorname{Im}\left[W^{k}(-i \overline{\mathcal{A}} \mathcal{B}+\bar{W})\right]}{|i \mathcal{A B}+W|^{2}} & =0 \\
-2 \frac{\operatorname{Re} Y_{\tau}}{Y}+(2 \Gamma-1) \frac{\operatorname{Re} \mathcal{B}_{\tau}}{\mathcal{B}}+\frac{c}{3} \mathcal{B} \frac{\mathcal{A}_{1} \mathcal{B}+\operatorname{Im} W}{\left(\mathcal{A}_{1} \mathcal{B}+\operatorname{Im} W\right)^{2}+\left(-\mathcal{A}_{2} \mathcal{B}+\operatorname{Re} W\right)^{2}} & =0 \\
-2 \frac{\operatorname{Im} Y_{\tau}}{Y}+(2 \Gamma-1) \frac{\operatorname{Im} \mathcal{B}_{\tau}}{\mathcal{B}}+\frac{c}{3} \mathcal{B} \frac{A_{2} \mathcal{B}+\operatorname{Re} W}{\left(\mathcal{A}_{1} \mathcal{B}+\operatorname{Im} W\right)^{2}+\left(-\mathcal{A}_{2} \mathcal{B}+\operatorname{Re} W\right)^{2}} & =-\frac{1}{2 \tau_{2}}(A)
\end{aligned}
$$

Note first that the middle two real equations $\operatorname{Im} G_{k}=0$ and $\operatorname{Re} G_{\tau}=0$, which have a zero on the right hand side, have a major part in common (again $W$ denotes $W^{(\mathcal{T})}$ )

$$
\begin{aligned}
& \operatorname{Im} \tilde{C}_{k}\left[\frac{2}{Y+2 \tilde{C}}+(2 \Gamma-1) \frac{2 \tilde{C}}{Y+2 \tilde{C}} \frac{1}{Y+\tilde{C}}\right]=a_{k} \frac{\operatorname{Im}\left(W^{k}(-i \overline{\mathcal{A}} \mathcal{B}+\bar{W})\right)}{|i \mathcal{A B}+W|^{2}} \\
& \operatorname{Re} \tilde{C}_{\tau}\left[\frac{2}{Y+2 \tilde{C}}+(2 \Gamma-1) \frac{2 \tilde{C}}{Y+2 \tilde{C}} \frac{1}{Y+\tilde{C}}\right]=-\frac{c}{3} \mathcal{B} \frac{\mathcal{A}_{1} \mathcal{B}+\operatorname{Im} W}{|i \mathcal{A B}+W|^{2}}
\end{aligned}
$$

Using (A.11) in (A.10) gives

$-\frac{c}{3} \mathcal{B} \frac{\operatorname{Im} \tilde{C}_{k}}{\operatorname{Re} \tilde{C}_{\tau}}\left(\mathcal{A}_{1} \mathcal{B}+\operatorname{Im} W\right)=a_{k}\left(-\operatorname{Re} W^{k}\left(\mathcal{A}_{1} \mathcal{B}+\operatorname{Im} W\right)+\operatorname{Im} W^{k}\left(-\mathcal{A}_{2} \mathcal{B}+\operatorname{Re} W\right)\right)($

Dividing by $a_{k}$ and summing over $k$ one arrives at

$$
\frac{-c \mathcal{B}}{\operatorname{Re} \tilde{C}_{\tau}} \frac{\mathcal{A}_{1} \operatorname{Re} W+\mathcal{A}_{2} \operatorname{Im} W}{2 A \bar{A}}\left(\mathcal{A}_{1} \mathcal{B}+\operatorname{Im} W\right)=-\mathcal{B}\left(\mathcal{A}_{1} \operatorname{Re} W+\mathcal{A}_{2} \operatorname{Im} W\right)
$$

This implies $\mathcal{A}_{1} \operatorname{Re} W+\mathcal{A}_{2} \operatorname{Im} W=0$ for otherwise one would get $\mathcal{B}=2 \frac{\mathcal{A}_{2} \operatorname{Re} W-\mathcal{A}_{1} \operatorname{Im} W}{\mathcal{A}_{1}^{2}+\mathcal{A}_{2}^{2}}$ from

$$
\left(\mathcal{A}_{1} \mathcal{B}+\operatorname{Im} W\right)\left(\mathcal{A}_{1}^{2}+\mathcal{A}_{2}^{2}\right)=2 \mathcal{A}_{1} \mathcal{A}_{2} \operatorname{Re} W-\left(\mathcal{A}_{1}^{2}-\mathcal{A}_{2}^{2}\right) \operatorname{Im} W
$$

But one has that $\mathcal{B}=Y-\frac{\mathcal{A}_{2} \operatorname{Re} W-\mathcal{A}_{1} \operatorname{Im} W}{\mathcal{A}_{1}^{2}+\mathcal{A}_{2}^{2}}$; this would then give $Y=3 \frac{\mathcal{A}_{2} \operatorname{Re} W-\mathcal{A}_{1} \operatorname{Im} W}{\mathcal{A}_{1}^{2}+\mathcal{A}_{2}^{2}}=$ $-3 \tilde{C}$ which is excluded from (6.15) as $\xi \neq 0$. So the remaining possibility is $\operatorname{Im} W=$ $-\frac{\mathcal{A}_{1}}{\mathcal{A}_{2}} \operatorname{Re} W$ and so $\tilde{C}=-\frac{\operatorname{Re} W}{\mathcal{A}_{2}}$. Applying now (A.11) in (A.9) would give finally $0=$ $-\frac{1}{2 \tau_{2}} \frac{3}{c \mathcal{B}} \frac{\mathcal{A}_{1}^{2}+\mathcal{A}_{2}^{2}}{\mathcal{A}_{2}}\left(\mathcal{A}_{2} \mathcal{B}+\operatorname{Re} W\right) \frac{\mathcal{A}_{1}}{\mathcal{A}_{2}} \operatorname{Re} W$, so $\mathcal{A}_{1}=0$ or $\operatorname{Re} W=0$ (and so in any case $\operatorname{Im} W=0$ ); however above we had assumed that $\operatorname{Re} \tilde{C}_{\tau} \neq 0$ and so this can not be argued.

Rather one has from $\operatorname{Im} W=-\frac{\mathcal{A}_{1}}{\mathcal{A}_{2}} \operatorname{Re} W$ and $\tilde{C}=-\frac{\operatorname{Re} W}{\mathcal{A}_{2}}$ (still assuming $\operatorname{Re} \tilde{C}_{\tau} \neq 0$ ) that (A.9) gives finally $4 \mathcal{A}_{2}^{2} \mathcal{B}(\operatorname{Re} W)^{2}=\left(\mathcal{B}+2 \mathcal{A}_{2} \operatorname{Re} W\right)\left(\left(\mathcal{A}_{2} \mathcal{B}\right)^{2}-(\operatorname{Re} W)^{2}\right)$ or

$$
Y^{3}+\left(3 \tilde{C}-2 \mathcal{A}_{2}^{2} \tilde{C}\right) Y^{2}+2\left(1-4 \mathcal{A}_{2}^{2}\right) \tilde{C}^{2} Y+4 \mathcal{A}_{2}^{2} \cdot \tilde{C}^{3}=0
$$

Combining with (6.15) one gets eliminations with which $G_{k}=0$ can be treated further. 


\section{References}

1. S.B. Giddings, S. Kachru and J. Polchinski, Hierarchies from Fluxes in String Compactifications, hep-th/0105097, Phys. Rev. D66 (2002) 106006.

2. S. Gukov, C. Vafa and E. Witten, CFT's From Calabi-Yau Four-folds, hepth/9906070, Nucl.Phys. B584 (2000) 69, Erratum-ibid. B608 (2001) 477;

S. Gukov, Solitons, Superpotentials and Calibrations, hep-th/9911011, Nucl.Phys. B574 (2000) 169;

T. R. Taylor and C. Vafa, RR flux on Calabi-Yau and partial supersymmetry breaking, Phys. Lett. B 474, 130 (2000), hep-th/9912152;

P. Mayr, On supersymmetry breaking in string theory and its realization in brane worlds, Nucl. Phys. B 593, 99 (2001), hep-th/0003198;

G. Curio, A. Klemm, D. Lüst and S. Theisen, On the vacuum structure of type II string compactifications on Calabi-Yau spaces with H-fluxes, Nucl. Phys. B 609, 3 (2001), hep-th/0012213.

3. S. Kachru, R. Kallosh, A. Linde and S.P. Trivedi, De Sitter Vacua in String Theory, hep-th/0301240, Phys. Rev. D68 (2003) 046005.

4. H.P. Nilles, Gaugino Condensation and SUSY Breakdown, hep-th/0402022;

S. Ferrara, L. Girardello and H.P. Nilles, Breakdown Of Local Supersymmetry Through Gauge Fermion Condensates, Phys. Lett. B125 (1983) 457;

M. Dine, R. Rohm, N. Seiberg and E. Witten, Gluino Condensation in Superstring Models, Phys. Lett. B156 (1985) 55;

J.P. Derendinger, L. E. Ibanez and H. P. Nilles, On The Low-Energy $D=4, N=1$ Supergravity Theory Extracted From The $D=10$, N=1 Superstring, Phys. Lett. B155 (1985) 65;

J.P. Derendinger, L.E. Ibanez and H. P. Nilles, On The Low-Energy Limit Of Superstring Theories, Nucl. Phys. B267 (1986) 365;

A. Font, L.E. Ibanez, D. Lüst and F. Quevedo, Supersymmetry Breaking From Duality Invariant Gaugino Condensation, Phys. Lett. B245 (1990) 401;

S. Ferrara, N. Magnoli, T.R. Taylor and G. Veneziano, Duality And Supersymmetry Breaking In String Theory, Phys. Lett. B245 (1990) 409;

H.P. Nilles and M. Olechowski, Gaugino Condensation And Duality Invariance, Phys. Lett. B248 (1990) 268.

5. E. Witten, Non-Perturbative Superpotentials In String Theory, hep-th/9604030, Nucl.Phys. B474 (1996) 343. 
6. L. Goerlich, S. Kachru, P.K. Tripathy and S.P. Trivedi, Gaugino Condensation and Nonperturbative Superpotentials in Flux Compactifications, hep-th/0407130, JHEP 0412 (2004) 074;

R. Kallosh and D. Sorokin, Dirac Action on M5 and M2 Branes with Bulk Fluxes, hep-th/0501081, JHEP 0505 (2005) 005;

P.K. Tripathy and S.P. Trivedi, D3 Brane Action and Fermion Zero Modes in Presence of Background Flux, hep-th/0503072, JHEP 0506 (2005) 066;

E. Bergshoeff, R. Kallosh, A.-K. Kashani-Poor, D. Sorokin and A. Tomasiello, An index for the Dirac operator on D3 branes with background fluxes, hep-th/0507069, JHEP 0510 (2005) 102;

R. Kallosh, A.-K. Kashani-Poor and A. Tomasiello, Counting fermionic zero modes on M5 with fluxes, hep-th/0503138, JHEP 0506 (2005) 069;

D. Lust, S. Reffert, W. Schulgin and P. Tripathy, Fermion Zero Modes in the Presence of Fluxes and a Non-perturbative Superpotential, hep-th/0509082.

7. K. Choi, A. Falkowski, H. P. Nilles, M. Olechowski and S. Pokorski, Stability of Flux Compactifications and the Pattern of Supersymmetry Breaking, hep-th/0411066, JHEP 0411 (2004) 076.

8. S.P. de Alwis, Effective Potentials for Light Moduli, Phys.Lett. B626 (2005) 223, hep-th/0506266.

9. Frederik Denef, Michael R. Douglas, Bogdan Florea, Building a Better Racetrack, hep-th/0404257, JHEP 0406 (2004) 034.

10. J.J. Blanco-Pillado, C.P. Burgess, J.M. Cline, C. Escoda, M. Gomez-Reino, R. Kallosh, A. Linde and F. Quevedo, Inflating in a Better Racetrack, hep-th/0603129.

11. J.P. Conlon and F. Quevedo, Gaugino and Scalar Masses in the Landscape, hepth/0605141.

12. V. Balasubramanian, P. Berglund, J.P. Conlon and F. Quevedo, Systematics of Moduli Stabilisation in Calabi-Yau Flux Compactifications, hep-th/0502058, JHEP 0503 (2005) 007.

13. A. Grassi, Divisors on elliptic Calabi-Yau 4-folds and the superpotential in F-theory, I, alg-geom/9704008.

14. S. Katz and C. Vafa, Geometric Engineering of $N=1$ Quantum Field Theories, hep-th/9611090, Nucl.Phys. B497 (1997) 196. 
15. R. Friedman, J. Morgan and E. Witten, Vector Bundles And F Theory, hepth/9701162, Commun.Math.Phys. 187 (1997) 679.

16. P. Candelas, A. Font, S. Katz and D.R. Morrison, Mirror Symmetry for Two Parameter Models - II, hep-th/9403187, Nucl.Phys. B429 (1994) 626.

17. J. Wess and J. Bagger, "Supersymmetry and Supergravity", Second Edition, Revised and Expanded (1992), Princeton Series in Physics, Princeton University Press, Princeton, New Jersey.

18. M. Becker, G. Curio and A. Krause, De Sitter Vacua from Heterotic M-Theory, hep-th/0403027, Nucl.Phys. B693 (2004) 223.

19. G. Curio and A. Krause, Moduli Stabilization in New Heterotic de Sitter Vacua, to appear.

20. G. Curio, A. Krause and D. Lust, Moduli Stabilization in the Heterotic/IIB Discretuum, hep-th/0502168, Fortsch.Phys. 54 (2006) 225. 